\title{
REMARKS ON MOTIVIC MOORE SPECTRA
}

\author{
OLIVER RÖNDIGS
}

\begin{abstract}
The term "motivic Moore spectrum" refers to a cone of an element $\alpha: \Sigma^{s, w} \mathbf{1} \rightarrow \mathbf{1}$ in the motivic stable homotopy groups of spheres. Homotopy groups, multiplicative structures, and Voevodsky's slice spectral sequence are discussed for motivic Moore spectra.
\end{abstract}

\section{INTRODUCTION}

Let $R$ be a ring and $a \in R$ an element, generating a two-sided ideal $(a) \subset R$. The projection onto the quotient $R \rightarrow R / a:=R /(a)$ is then a ring homomorphism. If $R$ is commutative, then so is $R / a$. In homotopy theory, the situation is more subtle. The topological sphere spectrum $\mathbb{S}$ is the unit in a closed symmetric monoidal category modeling the stable homotopy category, and in particular a commutative monoid. Given any endomorphism $a: \mathbb{S} \rightarrow \mathbb{S}$, the homotopy-theoretic quotient is almost never a commutative monoid. The first instance occurs when $a=2$ : The homotopy-theoretic quotient $\mathbb{S} / 2$, also known as the Moore spectrum for the group $\mathbb{Z} / 2$, satisfies $\pi_{2} \mathbb{S} / 2 \cong \mathbb{Z} / 4$ by [3], and in particular admits no unital multiplicative structure. Even if a unital multiplicative structure exists on $\mathbb{S} / n$ for $n>2$, its associativity or commutativity in the stable homotopy category is not automatic. See 16 and the references therein for details.

Within motivic or $\mathbf{A}^{1}$-homotopy theory, Moore spectra have appeared for example in [18, 6], and [11. As the structure of the endomorphisms of the motivic sphere spectrum is much richer, also the notion of Moore spectra should be more sophisticated. The degree zero part of these endomorphisms over a field is the Milnor-Witt $K$-theory graded by weight [14, and the weight zero part of that is the Grothendieck-Witt ring of the field. Since neither is a principal ideal domain in general, and usually far from Noetherian, Moore spectra with respect to ideals instead of single elements are more sensible. See for example [11, Remark 1.4]. Nevertheless, an elementary approach is chosen here, which still suffices to illustrate a few interesting phenomena. More precisely, multiplicative structures on motivic Moore spectra - whose existence may depend on the ground field - are discussed in Section [5, based to some extent on results concerning Toda brackets listed in Section 4. These in turn rely on some preliminaries on the few first stable stems of the motivic sphere spectrum, to be discussed in Section 2, which follows and partly expands 21. The article closes with some results on slices and slice differentials for special motivic Moore spectra in Section 6. These results may be used for slice spectral sequence computations of homotopy groups of motivic Moore spectra. A

Date: October 3, 2019.

This work was supported through DFG grants within the SPP 1786 "Homotopy theory and algebraic geometry", and a guest professorship at the University of Oslo. 
noteworthy feature in comparison with corresponding slice spectral sequence computations for the motivic sphere spectrum is the absence of motivic cohomology groups with integral coefficients; motivic cohomology groups with finite coefficients are understood much better.

\section{Preliminaries on $\pi_{0}, \pi_{1}$, AND $\pi_{2}$}

Determining the existence of multiplications or pairings on Moore spectra requires information about stable homotopy groups of motivic spheres. Let $\pi_{s, w} \mathrm{E}$ denote the abelian group $\left[\Sigma^{s, w} \mathbf{1}, \mathrm{E}\right]$, where $\mathrm{E}$ is a motivic spectrum and $\mathbf{1}$ is the motivic sphere spectrum. Set $\pi_{s+(w)} \mathrm{E}:=\pi_{s+w, w} \mathrm{E}$, and let

$$
\pi_{s+(\star)} \mathrm{E}=\bigoplus_{w \in \mathbb{Z}} \pi_{s+w, w} \mathrm{E}
$$

denote the direct sum, considered as a $\mathbb{Z}$-graded module over the $\mathbb{Z}$-graded ring $\pi_{0+(\star)}$ 1. The notation $\pi_{s-(\star)} \mathrm{E}:=\pi_{s+(-\star)} \mathrm{E}$ will be used frequently. The strictly $\mathbf{A}^{1}$-invariant sheaf obtained as the associated Nisnevich sheaf of $U \mapsto \pi_{s, w} \mathrm{E}_{U}$ for $U \in \mathbf{S m}_{F}$ is denoted $\underline{\pi}_{s, w} \mathrm{E}$, which gives rise to $\underline{\pi}_{s+(\star)} \mathrm{E}$. See [14] for the following statement.

Theorem 2.1 (Morel). Let $F$ be a field. Then $\pi_{0-(\star)} \mathbf{1}$ is the Milnor-Witt $K$-theory of $F$.

The Milnor-Witt $K$-theory of $F$ is denoted $\mathbf{K}^{\mathrm{MW}}(F)$, or simply $\mathbf{K}^{\mathrm{MW}}$, following the convention that the base field or scheme may be ignored in the notation. Its generators are denoted $\eta \in \mathbf{K}_{-1}^{\mathrm{MW}}=\pi_{1,1} \mathbf{1}$ and $[u] \in \mathbf{K}_{1}^{\mathrm{MW}}(F)=\pi_{-1,-1} \mathbf{1}_{F}$ for every unit $u \in F^{\times}$. The abbreviations

$$
\begin{aligned}
\langle u\rangle & :=1+\eta[u] \in \mathbf{K}_{0}^{\mathrm{MW}}(F) \\
\left\langle u_{1}, \ldots, u_{m}\right\rangle & :=\left\langle u_{1}\right\rangle+\cdots+\left\langle u_{m}\right\rangle \in \mathbf{K}_{0}^{\mathrm{MW}}(F) \\
\varepsilon & :=-\langle-1\rangle \\
\mathrm{h} & :=\langle 1,-1\rangle=1-\varepsilon
\end{aligned}
$$

for units $u, u_{1}, \ldots, u_{m} \in F^{\times}$will be convenient. Under the identification of $\mathbf{K}_{0}^{\mathrm{MW}}(F)$ with the Grothendieck-Witt ring $\mathbf{G W}(F)$ of $F$, the element $\left\langle u_{1}, \ldots, u_{m}\right\rangle$ corresponds to the quadratic form given by the appropriate diagonal matrix. Milnor $K$-theory [12] is expressed as the quotient $\mathbf{K}_{\star}^{\mathrm{M}} \cong \mathbf{K}_{\star}^{\mathrm{MW}} /(\eta)$. Set $\mathbf{k}_{\star}^{\mathrm{M}}:=\mathbf{K}_{\star}^{\mathrm{M}} / 2$. Theorem 2.1 implies that for every motivic spectrum $\mathrm{E}$ and for every integer $s, \pi_{s+(\star)} \mathrm{E}$ is a graded $\mathbf{K}^{\mathrm{MW}}$-module. As a first instance besides the motivic sphere spectrum $\mathbf{1}$, consider the very effective cover $\mathrm{kq} \rightarrow \mathrm{KQ}$ of the motivic spectrum representing hermitian $K$-theory [1], [2]. Using kq instead of the effective cover $\mathrm{f}_{0} \mathrm{KQ} \rightarrow \mathrm{KQ}$ leads to a slight improvement on the computation [21, Theorem 5.5].

Theorem 2.2 (Röndigs-Spitzweck-Østvær). Let $F$ be a field of exponential characteristic $e \neq 2$. The unit map $\mathbf{1} \rightarrow \mathrm{kq}$ induces an isomorphism $\pi_{0+(\star)} \mathbf{1} \rightarrow \pi_{0+(\star)} \mathrm{kq}$, and a surjection $\pi_{1+(\star)} \mathbf{1} \rightarrow \pi_{1+(\star)} \mathrm{kq}$ whose kernel coincides with $\mathbf{K}_{2-\star}^{\mathrm{M}} / 24$ after inverting $e$.

Voevodsky's slice filtration $\left\{\mathrm{f}_{q} \mathrm{E} \rightarrow \mathrm{E}\right\}_{q \in \mathbb{Z}}$ allows to be more precise, and in particular to describe the $\mathbf{K}^{\mathrm{MW}}$-module structure. Let $\mathbf{M} \mathbb{Z}$ be Voevdosky's integral motivic Eilenberg-MacLane spectrum representing motivic cohomology, and 
let $\mathbf{M} \mathbb{Z} / 2$ be the version with coefficients in $\mathbb{Z} / 2$. Moreover, set for $k$ a natural number $H^{\star-k, \star}:=\pi_{k-(\star)} \mathbf{M} \mathbb{Z}$ and $h^{\star-k, \star}:=\pi_{k-(\star)} \mathbf{M} \mathbb{Z} / 2$. Note the $\mathbf{K}^{\mathrm{MW}}$-module isomorphism $h^{\star-k, \star} \cong \mathbf{k}_{\star-k}^{\mathrm{M}}$ given by multiplication with $\tau^{k}$, where

$$
\tau=-1 \in h^{0,1}=\{1,-1\}=\operatorname{ker}\left(H^{1,1} \stackrel{x \mapsto x^{2}}{\longrightarrow} H^{1,1}\right)
$$

is the unique nontrivial element. The $\mathbf{K}^{\mathrm{MW}}$-module $\pi_{1-(\star)} \mathrm{kq}$ is an extension of the $\mathbf{K}^{\mathrm{MW}}$-module $H^{\star-1, \star}=\pi_{1-(\star)} \mathrm{s}_{0} \mathrm{kq}$ (on which $\eta$ operates trivially) and the $\mathbf{K}^{\mathrm{MW}}$ module given by the image of $\pi_{1-(\star)} \mathrm{f}_{1} \mathrm{kq}$ in $\pi_{1-(\star)} \mathrm{kq}$.

Lemma 2.3. The $\mathbf{K}^{\mathrm{MW}}{ }_{- \text {module }} \pi_{1-(\star)} \mathrm{f}_{1} \mathrm{kq}$ is generated by the image of $\eta_{\text {top }}$ under the unit map $\mathrm{u}: \mathbf{1} \rightarrow \mathrm{kq}$, and has the presentation

$$
\mathbf{K}^{\mathrm{MW}} /\left(2, \eta^{2}\right) \cong \pi_{1-(\star)} \mathrm{f}_{1} \mathrm{kq} .
$$

Proof. The determination of the relevant part of the slice spectral sequence for $\mathrm{kq}$ given in [1, Proposition 27] implies that the $\mathbf{K}^{\mathrm{MW}}$-module $\pi_{1-(\star)} \mathrm{f}_{1} \mathrm{kq}$ is an extension of the $\mathbf{K}^{\mathrm{MW}}$-module $h^{\star, 1+\star}=\pi_{1-(\star)} \mathrm{s}_{1} \mathrm{kq}$ (on which $\eta$ operates trivially) and the $\mathbf{K}^{\mathrm{MW}}$-module $h^{1+\star, 2+\star} / \mathrm{Sq}^{2} h^{\star-1,1+\star}$ (on which $\eta$ operates trivially as well). The $\mathbf{K}^{\mathrm{M}}$-module $h^{\star, 1+\star}=\pi_{1-(\star)} \mathrm{s}_{1} \mathrm{kq}$ is generated by the image of $\eta_{\text {top }} \in \pi_{1,0} \mathbf{1}$, and the $\mathbf{K}^{\mathrm{M}}$-module $h^{1+\star, 2+\star} / \mathrm{Sq}^{2} h^{\star-1,1+\star}$ is generated by the image of $\eta \eta_{\text {top }} \in \pi_{2,1} \mathbf{1}$. Since $2 \eta_{\text {top }}=0$, the extension describing $\pi_{1-(\star)} f_{1} \mathrm{kq}$ splits in every degree as a short exact sequence of abelian groups. In other words, the surjection $\mathbf{K}^{\mathrm{MW}} \rightarrow \pi_{1-(\star)} \mathrm{kq}$ factors through a surjection $\mathbf{K}^{\mathrm{MW}} / 2 \rightarrow \pi_{1-(\star)} \mathrm{f}_{1} \mathrm{kq}$. The relation $0=\eta^{2} \mathrm{u}\left(\eta_{\text {top }}\right) \in \pi_{3,2} \mathrm{f}_{1} \mathrm{kq}$ follows from the slice spectral sequence computation (which even gives $\pi_{3,2} \mathrm{kq}=0$ ).

In order to show that the obtained surjection $\mathbf{K}^{\mathrm{MW}} /\left(2, \eta^{2}\right) \rightarrow \pi_{1-(\star)} f_{1} \mathrm{kq}$ is an isomorphism, observe that it fits into a natural transformation

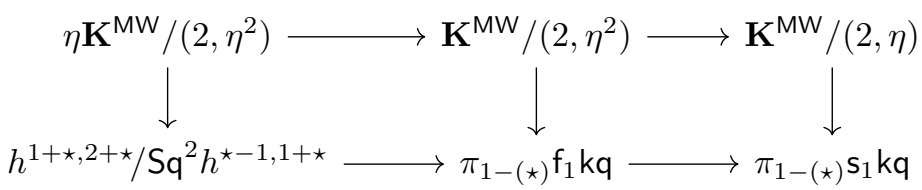

of short exact sequences, where the outer vertical morphisms are isomorphisms. This implies the result.

The difference between $\pi_{1-(\star)} f_{1} \mathrm{kq}$ and its image $f_{1} \pi_{1-(\star)} \mathrm{kq}$ in $\pi_{1-(\star)} \mathrm{kq}$ is given by the image of $\pi_{2-(\star)} \mathrm{s}_{0} \mathrm{kq} \cong H^{\star-2, \star} \rightarrow \pi_{1-(\star)} \mathrm{f}_{1} \mathrm{kq}$. Since $2 \eta_{\text {top }}=0$, this map factors over $H^{\star-2, \star} / 2$. The short exact sequence displayed in (2.1) then induces a long exact sequence

$\operatorname{Hom}_{\mathbf{k}^{\mathrm{M}}}\left(H^{\star-2, \star} / 2, \mathbf{k}_{\star}^{\mathrm{M}}{ }^{2} / \rho^{2}\right) \rightarrow \operatorname{Hom}_{\mathbf{K}^{\mathrm{MW}}}\left(H^{\star-2, \star} / 2, \pi_{1-(\star)} \mathrm{f}_{1} \mathbf{k q}\right) \rightarrow \operatorname{Hom}_{\mathbf{k}^{\mathrm{M}}}\left(H^{\star-2, \star} / 2, \mathbf{k}^{\mathrm{M}}\right) \rightarrow \cdots$ in which the first group is zero for any finite field or any number field. Here $\rho$ is the class of -1 in $h^{1,1}$. The homomorphism in question maps to the restriction of the motivic Steenrod square $\mathrm{Sq}^{2}$ in the abelian group $\operatorname{Hom}_{\mathbf{k}^{\mathrm{M}}}\left(H^{\star-2, \star} / 2, h^{\star, \star+1} \cong \mathbf{k}_{\star}^{\mathrm{M}}\right)$, and is thereby uniquely determined for prime fields.

Following a specific request, a probably well-known identification, in which $\mathcal{O}^{\times}$ denotes the sheaf of units, can be derived from Lemma 2.3. In principle, any sequence of strictly $\mathbf{A}^{1}$-invariant sheaves which is exact when evaluated on fields is already exact, by a theorem of Morel. This applies in particular to Theorem 2.2 , Lemma 2.3, and Theorem 2.5. However, the following case can be proved directly instead. 
Proposition 2.4. Let $F$ be a field of characteristic not two. The sheaf $\underline{\pi}_{1,0} \mathrm{KQ}$ is isomorphic to the sheaf $\mathcal{O}^{\times} / 2 \times \mathbb{Z} / 2$.

Proof. By construction, the canonical maps induce isomorphisms $\underline{\pi}_{1,0} \mathrm{f}_{1} \mathrm{kq} \cong \underline{\pi}_{1,0} \mathrm{kq} \cong$ $\underline{\pi}_{1,0} \mathrm{KQ}$. This uses the vanishing $\underline{\pi}_{1,0} \mathrm{~s}_{0} \mathrm{kq}=\underline{\pi}_{2,0} \mathrm{~s}_{0} \mathrm{kq}=0$, since $\mathrm{s}_{0} \mathrm{kq}=\mathbf{M} \mathbb{Z}$ and motivic cohomology of smooth schemes in weight zero is concentrated in degree zero. The homotopy cofiber sequence

$$
\mathrm{f}_{2} \mathrm{kq} \rightarrow \mathrm{f}_{1} \mathrm{kq} \rightarrow \mathrm{s}_{1} \mathrm{kq}
$$

induces, for every smooth connected $F$-scheme $U$, a short exact sequence

$0=\pi_{2,0} \mathrm{~s}_{1} \mathrm{kq}(U) \rightarrow \pi_{1,0} \mathrm{f}_{2} \mathrm{kq}(U) \rightarrow \pi_{1,0} \mathrm{f}_{1} \mathrm{kq}(U) \rightarrow \pi_{1,0} \mathrm{~s}_{1} \mathrm{kq}(U)=h^{0,1}(U)=\mathbb{Z} / 2 \rightarrow 0$

which splits naturally via the map $\mathbb{Z} / 2=\pi_{1} \mathbb{S} \rightarrow \pi_{1,0} \mathrm{f}_{1} \mathrm{kq}$. In case $U$ is (the spectrum of) a field extension, this sequence appears in diagram (2.1). Already if $U$ is an essentially smooth local $F$-scheme, then $\pi_{1,0} \mathrm{f}_{2} \mathrm{kq}(U) \cong \pi_{1,0} \mathrm{~s}_{2} \mathrm{kq}(U) \cong h^{1,2}(U)$ which supplies the result on the level of Nisnevich sheaves.

Theorem 2.5. Let $\nu \in \pi_{3,2} 1$ be the second algebraic Hopf map, obtained by the Hopf construction on $\mathbf{S L}_{2}$, and let $\eta_{\text {top }} \in \pi_{1,0} \mathbf{1}$ be the first topological Hopf map. The $\mathbf{K}^{\mathrm{MW}}$-module map

$$
\mathbf{K}_{2+\star}^{\mathrm{MW}} \oplus \mathbf{K}^{\mathrm{MW}} \rightarrow \pi_{1-(\star)} \mathrm{f}_{1} \mathbf{1}
$$

sending $(a, b)$ to $a \cdot \nu+b \cdot \eta_{\text {top }}$ induces an isomorphism

$$
\mathbf{K}_{2+\star}^{\mathrm{MW}}\{\nu\} \oplus \mathbf{K}^{\mathrm{MW}}\left\{\eta_{\text {top }}\right\} /\left(\eta \nu, 2 \eta_{\text {top }}, \eta^{2} \eta_{\text {top }}-12 \nu\right) \cong \pi_{1-(\star)} \mathrm{f}_{1} \mathbf{1}
$$

after inverting the exponential characteristic.

Proof. Observe first that $\nu$ naturally lifts to $\pi_{3,2} f_{2} \mathbf{1}$, hence defines also an element $\nu \in \pi_{3,2} \mathrm{f}_{1} \mathbf{1}$. The element $\eta_{\text {top }}$ has image $0 \in \pi_{1,0} \mathrm{~s}_{0} \mathbf{1}=\pi_{1,0} \mathbf{M} \mathbb{Z}=H^{-1,0}$, and hence also lifts to $\pi_{1,0} \mathrm{f}_{1} \mathbf{1}$ - even uniquely, since $\pi_{2,0} \mathrm{~s}_{0} \mathbf{1}=\pi_{2,0} \mathbf{M} \mathbb{Z}=H^{-2,0}=0$. Thus the $\mathbf{K}^{\mathrm{MW}}$-module map

$$
\mathbf{K}_{2+\star}^{\mathrm{MW}} \oplus \mathbf{K}^{\mathrm{MW}} \rightarrow \pi_{1-(\star)} \mathrm{f}_{1} \mathbf{1}
$$

sending $(a, b)$ to $a \cdot \nu+b \cdot \eta_{\text {top }}$ is well-defined over any base scheme in which motivic cohomology in weight zero vanishes in degree -1 . Certain relations hold before inverting the exponential characteristic. The relation $\eta \nu=0$ holds by 4 over $\operatorname{Spec}(\mathbb{Z})$. Also $2 \eta_{\text {top }}=0$, again over any base scheme. If 2 is invertible in the base scheme, $\mathrm{u}(\nu)=0$, where $\mathrm{u}: \mathbf{1} \rightarrow \mathrm{kq}$ is the unit map figuring in Lemma 2.3.

Assuming that the exponential characteristic is now implicitly inverted, the exact sequence from Theorem 2.2 implies that $\pi_{3,2} \mathbf{1}=\mathbb{Z} / 24$, generated by $\nu$. Either by reference to complex realization or by the multiplicative structure of the slice spectral sequence (more precisely, only the effect of multiplying with the first algebraic Hopf map $\eta$ ), the element $\eta^{2} \eta_{\text {top }} \in \pi_{3,2} \mathbf{1}$ is the unique nontrivial element of order two, hence $\eta^{2} \eta_{\text {top }}=12 \nu=6 \mathrm{~h} \nu$. Thus there is an induced $\mathbf{K}^{\mathrm{MW}}$-module homomorphism

$$
\mathbf{K}_{2+\star}^{\mathrm{MW}}\{\nu\} \oplus \mathbf{K}^{\mathrm{MW}}\left\{\eta_{\text {top }}\right\} /\left(\eta \nu, 2 \eta_{\text {top }}, \eta^{2} \eta_{\text {top }}-12 \nu\right) \rightarrow \pi_{1-(\star)} \mathrm{f}_{1} \mathbf{1} .
$$

The $\mathbf{K}^{\mathrm{MW}}$-module homomorphism $\pi_{1-(\star)} \mathrm{f}_{1} \mathbf{1} \rightarrow \pi_{1-(\star)} \mathrm{f}_{1} \mathrm{kq}$ induced by the unit map $\mathrm{u}: \mathbf{1} \rightarrow \mathrm{kq}$ then factors over the isomorphism

$$
\mathbf{K}^{\mathrm{MW}} /\left(2, \eta^{2}\right) \cong \pi_{1-(\star)} \mathrm{f}_{1} \mathrm{kq}
$$


from Lemma 2.3. There results a commutative diagram

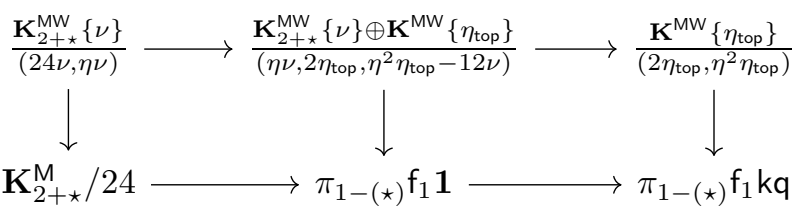

of short exact sequences, where the upper horizontal row is short exact by direct computation. Note that the relations $2 \eta_{\text {top }}=0$ and $\eta^{2} \eta_{\text {top }}=12 \nu$ imply $24 \nu=0$. The exactness of the lower horizontal row follows from the exactness of the sequence in Theorem 2.2 and the fact that the unit $\mathrm{u}: \mathbf{1} \rightarrow \mathrm{kq}$ induces an isomorphism on zero slices. The right vertical map in diagram (2.2) is an isomorphism by Lemma 2.3 . and the left vertical map in diagram (2.2) is an isomorphism by direct inspection, whence the result.

As in the case of $\mathrm{kq}$, the difference between $\pi_{1-(\star)} \mathrm{f}_{1} \mathbf{1}$ and its image $\mathrm{f}_{1} \pi_{1-(\star)} \mathbf{1}$ in $\pi_{1-(\star)} \mathbf{1}$ is given by the image of $\pi_{2-(\star)} \mathrm{s}_{0} \mathbf{1} \cong H^{\star-2, \star} \rightarrow \pi_{1-(\star)} \mathrm{f}_{1} \mathbf{1}$. The short exact sequence

$$
0 \rightarrow \mathrm{f}_{1} \pi_{1-(\star)} \mathbf{1} \rightarrow \pi_{1-(\star)} \mathbf{1} \rightarrow \pi_{1-(\star)} \mathrm{s}_{0} \mathbf{1}=H^{\star-1, \star} \rightarrow 0
$$

then describes the difference between the first two $\mathbf{K}^{\mathrm{MW}}$-modules. In particular, the canonical map $\pi_{1+w, w} \mathrm{f}_{1} \mathbf{1} \rightarrow \pi_{1+w, w} \mathbf{1}$ is an isomorphism for $w>-2$. For the applications to motivic Moore spectra, an important weight is $w=0$, where a short exact sequence

$$
0 \rightarrow \mathbf{K}_{2}^{\mathrm{M}} / 24 \rightarrow \pi_{1,0} \mathbf{1} \rightarrow \mathbb{Z} / 2 \oplus \mathbf{K}_{1}^{\mathrm{M}} / 2 \rightarrow 0
$$

results. One result of Theorem 2.5 is that the sequence (2.3) splits as a sequence of abelian groups, but not as a sequence of $\mathbf{K}_{0}^{\mathrm{MW}}$-modules. In particular, $24 \cdot \pi_{1,0} \mathbf{1}=0$, in contrast with $24 \cdot \pi_{-1,-2} \mathbf{1}_{\mathbb{Q}} \neq 0$, which is similar to $24 \cdot \pi_{-1,-2} \mathrm{KGL}_{\mathbb{Q}} \cong 24 \cdot \mathbb{Z} / 48 \neq$ 0 .

Lemma 2.6. The action of $\mathbf{G W}$ on $\pi_{1+(\star)} \mathbf{1}$ is determined by the following equations for $u \in F^{\times}$.

$$
\begin{aligned}
\langle u\rangle \cdot \nu & =\nu \in \pi_{3,2} \mathbf{1} \\
\langle u\rangle \cdot \eta \eta_{\text {top }} & =\eta \eta_{\text {top }}+\left[u^{12}\right] \nu \in \pi_{2,1} \mathbf{1} \\
\langle u\rangle \cdot \eta_{\text {top }} & =\eta_{\text {top }}+[u] \eta \eta_{\text {top }} \in \pi_{1,0} \mathbf{1}
\end{aligned}
$$

In particular, $\mathrm{h} \cdot \eta_{\text {top }}=\rho \eta \eta_{\text {top }}, \mathrm{h} \cdot \eta \eta_{\text {top }}=0$, and $\mathrm{h} \cdot \nu=2 \nu$. If $n$ is even, then $n \mathrm{~h}$ acts on $\pi_{1+(*)} \mathrm{f}_{1} \mathbf{1}$ as multiplication by $2 n$.

Proof. This follows from Theorem 2.5, once the identification $\pi_{1+(w)} \mathrm{f}_{1} \mathbf{1}=\pi_{1+(w)} \mathbf{1}$ for $w>-2$ is deduced from vanishing results for integral motivic cohomology of fields in weight $<2$.

The situation for $\pi_{2+(\star)} \mathbf{1}$ is a bit more delicate. Nevertheless, the following can be read off from the slice spectral sequence.

Theorem 2.7. The element $\nu^{2}: \Sigma^{6,4} \mathbf{1} \rightarrow \mathbf{1}$ induces an inclusion

$$
\mathbf{K}_{4+\star}^{\mathrm{MW}}\left\{\nu^{2}\right\} /\left(\eta \nu^{2}, 2 \nu^{2}\right) \rightarrow \pi_{2-(\star)} \mathbf{1}
$$

of $\mathbf{K}^{\mathrm{MW}}$-modules, which is an isomorphism for all $\star<-1$. In particular, for all $w>4$, the group $\pi_{2+w, w} \mathbf{1} \cong 0$. 
Proof. Since $\eta \nu=0$ [4, also $\eta \nu^{2}=0$. Moreover, the $\varepsilon$-graded commutativity of $\pi_{*+(\star)} \mathbf{1}$ implies that $\nu^{2}=-\nu^{2}$, whence a map $\mathbf{K}_{4+\star}^{\mathrm{MW}}\left\{\nu^{2}\right\} /\left(\eta \nu^{2}, 2 \nu^{2}\right) \rightarrow \pi_{2-(\star)} \mathbf{1}$ of $\mathrm{K}^{\mathrm{MW}}$-modules exists. The slice spectral sequence for $\pi_{2+(\star)} \mathbf{1}$ shows its injectivity, as well as the isomorphism statement, using results from [21] and [17, Theorem 8.3]; details are to be given in [20].

\section{Elementary properties of motivic Moore spectra}

Definition 3.1. Let $s \geq w \in \mathbb{Z}$, and let $\alpha: \Sigma^{s, w} \mathbf{1} \rightarrow \mathbf{1}$ be an endomorphism. Choosing a homotopy cofiber sequence

$$
\Sigma^{s, w} \mathbf{1} \stackrel{\alpha}{\rightarrow} \mathbf{1} \stackrel{c}{\rightarrow} \mathrm{C}_{\alpha} \stackrel{d}{\rightarrow} \Sigma^{s+1, w} \mathbf{1}
$$

defines the motivic Moore spectrum $\mathrm{C}_{\alpha}$.

By definition, a motivic Moore spectrum for $a$ is unique up to equivalence. The restriction $s \geq w$ is reasonable at least over a field by Morel's connectivity theorem [15.

Proposition 3.2. Let $\alpha: \Sigma^{s, w} \mathbf{1} \rightarrow \mathbf{1}$ be an endomorphism. The canonical maps induce a short exact sequence

$$
0 \rightarrow \pi_{n+(\star)} \mathbf{1} / \alpha \pi_{n-s+w+(\star-w)} \mathbf{1} \stackrel{c_{*}}{\longrightarrow} \pi_{n+(\star)} \mathrm{C}_{\alpha} \stackrel{d_{*}}{\longrightarrow}{ }_{\alpha} \pi_{n-1-s+w+(\star-w)} \mathbf{1} \rightarrow 0
$$

of $\mathbf{K}^{\mathrm{MW}}$-modules, with target the submodule of elements annihilated by $\alpha$.

Proof. This follows from the homotopy cofiber sequence

$$
\Sigma^{s, w} \mathbf{1} \stackrel{\alpha}{\rightarrow} \mathbf{1} \stackrel{c}{\rightarrow} \mathrm{C}_{\alpha} \stackrel{d}{\rightarrow} \Sigma^{s+1, w} \mathbf{1}
$$

defining the motivic Moore spectrum.

In particular, Morel's connectivity theorem implies with Proposition 3.2 that $\pi_{0+(\star)} \mathrm{C}_{\alpha} \cong \mathbf{K}^{\mathrm{MW}} / \alpha \mathbf{K}^{\mathrm{MW}}$ if $\alpha \in \mathbf{K}^{\mathrm{MW}}$ (that is, if $s=w$ ).

\section{TODA BRACKETS}

Consider three composable maps

$$
\mathrm{D} \stackrel{\gamma}{\rightarrow} \mathrm{E} \stackrel{\beta}{\rightarrow} \mathrm{F} \stackrel{\alpha}{\rightarrow} \mathrm{G}
$$

of motivic spectra such that $\beta \gamma=0=\alpha \beta$. The Toda bracket

$$
\langle\alpha, \beta, \gamma\rangle \quad\left(\bmod \alpha \circ\left[\Sigma^{1,0} \mathrm{D}, \mathrm{F}\right]+\left[\Sigma^{1,0} \mathrm{E}, \mathrm{G}\right] \circ \Sigma^{1,0} \gamma\right)
$$

is the coset of the displayed subgroup of $\left[\Sigma^{1,0} \mathrm{D}, \mathrm{G}\right]$ given by those compositions $\Sigma^{1,0} \mathrm{D} \rightarrow \mathrm{C}(\beta) \rightarrow \mathrm{G}$ such that the obvious diagrams commute. The base scheme or field may be indicated by a subscript. The most relevant case is where all the motivic spectra involved are appropriate suspensions of the motivic sphere spectrum 1. See the classical source [22, as well as [5] and [8] for interesting Toda brackets in the motivic stable homotopy category. We consider a few examples and make no claim to originality. Angled brackets are used to denote both Toda brackets and quadratic forms (as in the paragraph after Theorem 2.1). The reader should be aware of this possible confusion, but context will always make the meaning unambiguous. 
Proposition 4.1. The following equalities of subsets of $\pi_{*+(\star} 1$ hold.

$$
\begin{aligned}
\langle\mathrm{h}, \eta, \mathrm{h}\rangle & =\left\{\eta \eta_{\text {top }}+\left[\phi^{2}\right] \nu: \phi^{2} \in 2 \mathbf{K}_{1}^{\mathrm{M}}\{\nu\} / 24\right\} \\
\langle\eta, \mathrm{h}, \eta\rangle & =\{6 \nu,-6 \nu\} \\
\langle\eta, \nu, \eta\rangle & =\left\{\nu^{2}\right\} \\
\langle 2+11 \mathrm{~h}, \nu, 2+11 \mathrm{~h}\rangle_{\mathbb{R}} & =\left\{\rho^{2} \nu^{2}\right\}
\end{aligned}
$$

Proof. The indeterminacy in (4.1) is the subgroup $\mathrm{h} \circ \pi_{2,1} \mathbf{1}=2 \mathbf{K}_{1}^{\mathrm{M}}\{\nu\} / 24$, using

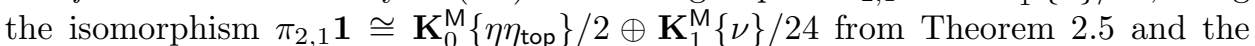
equalities $[-1] \eta\left(\eta \eta_{\text {top }}\right)=[-1] \eta^{2} \eta_{\text {top }}=[-1] 12 \nu=0=[-1] \eta \nu$ given in Lemma 2.6. That the Toda bracket contains $\eta \eta_{\text {top }}$ follows from appropriate realization functors and the equality $\left\langle 2, \eta_{\text {top }}, 2\right\rangle_{\text {top }}=\left\{\eta_{\text {top }}\right\}$ in classical stable homotopy groups 22 .

The indeterminacy in (4.2) is $\eta \circ \pi_{2,1}=\{0,12 \nu\}$, since Theorem 2.5 implies $\pi_{2,1} \mathbf{1} \cong \mathbf{K}_{0}^{\mathrm{M}}\left\{\eta \eta_{\text {top }}\right\} / 2 \oplus \mathbf{K}_{1}^{\mathrm{M}}\{\nu\} / 24$, and $\eta \eta \eta_{\text {top }}=12 \nu$, whereas $\eta \nu=0$. Complex realization maps $\langle\eta, \mathrm{h}, \eta\rangle$ to $\left\langle\eta_{\text {top }}, 2, \eta_{\text {top }}\right\rangle_{\text {top }}=\left\{6 \nu_{\text {top }},-6 \nu_{\text {top }}\right\}$. The relevant group $\pi_{3,2} \mathbf{1}$ does not depend on the base field (at least after inverting the exponential characteristic), giving the result.

The indeterminacy in (4.3) is the subgroup $\eta \circ \pi_{4,2}=\{0\}$, since $\pi_{4,2} \mathbf{1} \cong$ $\mathbf{K}_{2}^{\mathrm{M}}\left\{\nu^{2}\right\} / 2$ by Theorem 2.7, and $\eta \nu^{2}=0$. The unique element in $\langle\eta, \nu, \eta\rangle$ has to be the unique nontrivial one in $\pi_{6,4} \mathbf{1}=\mathbb{Z} / 2$ by appropriate realization.

Similar to the previous case, the indeterminacy in (4.4) is $\{0\}$ by Theorem 2.7 since $(2+11 \mathrm{~h}) \nu^{2}=0$. Real realization sends $\langle 2+11 \mathrm{~h}, \nu, 2+11 \mathrm{~h}\rangle_{\mathbb{R}}$ to $\left\langle 2, \eta_{\text {top }}, 2\right\rangle_{\text {top }}=$ $\left\{\eta_{\text {top }}^{2}\right\}$, whence the previous Toda bracket has to contain the unique nonzero element in $\pi_{4,2} \mathbf{1}_{\mathbb{R}}$.

The following statement, which could be formulated in greater generality, indicates the relevance of Toda brackets for the structure of motivic Moore spectra. The notation is as in Proposition 3.2 .

Proposition 4.2. Suppose $\alpha \in \pi_{s, w} 1, \beta \in \pi_{t, x} 1$, and $\gamma \in \pi_{u, y} 1$ are elements with $\alpha \beta=0=\beta \gamma$. The Toda bracket $\langle\alpha, \beta, \gamma\rangle$ coincides with the set of elements $\delta \in \pi_{s+t+u+1, w+x+y} \mathbf{1}$ for which there exists an element $\tilde{\beta} \in \pi_{s+t+1, w+x} \mathrm{C}_{\alpha}$ with $d_{*}(\tilde{\beta})=\beta$ and $c_{*}\left(\delta+\alpha \cdot \pi_{t+u+1, x+y} \mathbf{1}\right)=\tilde{\beta} \cdot \gamma$.

Proof. The proof consists of comparing various diagrams in the motivic stable homotopy category and is left as an exercise to the reader.

Example 4.3. Consider $\alpha=\gamma=\eta \in \pi_{1,1} 1$. Then $\pi_{5,3} \mathrm{C}_{\eta}$ contains an element $\tilde{\nu}$ with $\tilde{\nu} \cdot \eta \neq 0$. In fact, the Toda bracket $\langle\eta, \nu, \eta\rangle=\left\{\nu^{2}\right\}$ given in (4.3) contains a single nonzero element, and $c_{*}: \pi_{6,4} \mathbf{1} \rightarrow \pi_{6,4} \mathrm{C}_{\eta}$ is injective, since multiplication with $\eta$ is the zero map $\pi_{5,3} \mathbf{1}=h^{1,1}\left\{\nu^{2}\right\} \rightarrow h^{0,0}\left\{\nu^{2}\right\}=\pi_{6,4} \mathbf{1}$ by Theorem 2.7.

Example 4.4. Let $\alpha=\gamma=2+11 \mathrm{~h} \in \pi_{0,0} \mathbf{1}_{\mathbb{R}}$ (or any formally real field). Then $\pi_{4,2} \mathrm{C}_{2+11 \mathrm{~h}}$ contains an element $\tilde{\nu}$ with $\tilde{\nu} \cdot(2+11 \mathrm{~h}) \neq 0$. Indeed, the Toda bracket given in (4.4) contains a nonzero element (uniquely for $\mathbb{R}$ ), and $c_{*}: \pi_{4,2} \mathbf{1}_{\mathbb{R}} \rightarrow \pi_{4,2} \mathrm{C}_{2+11 \mathrm{~h}}$ is injective, since multiplication with $2+11 \mathrm{~h}$ induces the zero map $\pi_{4,2} \mathbf{1}_{\mathbb{R}}=h^{2,2}\left\{\nu^{2}\right\} \rightarrow \pi_{4,2} \mathbf{1}_{\mathbb{R}}$ by Theorem 2.7.

\section{Multiplications}

The "constant presheaf" functor defines a strict symmetric monoidal triangulated functor const: $\mathbf{S H} \rightarrow \mathbf{S H}(S)$ for any base scheme $S$. The motivic Moore 
spectra $C_{n}$ for $n \in \mathbb{N}$ are in its image. In particular, multiplications or pairings on these motivic Moore spectra can be transferred from the corresponding topological ones [16].

Lemma 5.1. Let $\alpha: \Sigma^{s, w} \mathbf{1} \rightarrow \mathbf{1}$ be an endomorphism and $c: \mathbf{1} \rightarrow \mathrm{C}_{\alpha}$ the map to the homotopy cofiber. There exists a left unital pairing

$$
\mathrm{C}_{\alpha} \wedge \mathrm{C}_{\alpha} \rightarrow \mathrm{C}_{\alpha}
$$

if and only if the identity on $\mathrm{C}_{\alpha}$ is annihilated by $\alpha$.

Proof. Consider the following diagram:

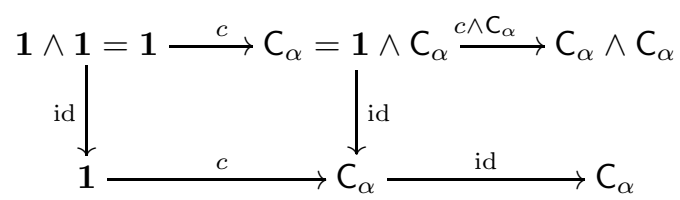

There exists a left unital pairing

$$
\mathrm{C}_{\alpha} \wedge \mathrm{C}_{\alpha} \rightarrow \mathrm{C}_{\alpha}
$$

if and only if the map $c \wedge C_{\alpha}$ admits a retraction. After smashing with $C_{\alpha}$, the homotopy cofiber sequence

$$
\Sigma^{s, w} \mathbf{1} \stackrel{\alpha}{\rightarrow} \mathbf{1} \stackrel{c}{\rightarrow} \mathrm{C}_{\alpha} \stackrel{d}{\rightarrow} \Sigma^{s+1, w} \mathbf{1}
$$

induces a long exact sequence

$$
\cdots \stackrel{d^{*}}{\longleftarrow}\left[\Sigma^{s, w} \mathrm{C}_{\alpha}, \mathrm{C}_{\alpha}\right] \stackrel{\alpha}{\leftarrow}\left[\mathrm{C}_{\alpha}, \mathrm{C}_{\alpha}\right] \stackrel{c^{*}}{\longleftarrow}\left[\mathrm{C}_{\alpha} \wedge \mathrm{C}_{\alpha}, \mathrm{C}_{\alpha}\right] \stackrel{d^{*}}{\longleftarrow}\left[\Sigma^{s+1, w} \mathrm{C}_{\alpha}, \mathrm{C}_{\alpha}\right] \stackrel{\alpha}{\leftarrow} \cdots
$$

which shows the desired statement.

If a left unital pairing $\mu: \mathrm{C}_{\alpha} \wedge \mathrm{C}_{\alpha} \rightarrow \mathrm{C}_{\alpha}$ exists, then $\mu \circ\left(\mathrm{C}_{\alpha} \wedge c\right) \circ c=c$. The short exact sequence

$$
0 \rightarrow \pi_{s+1, w} \mathrm{C}_{\alpha} \stackrel{d^{*}}{\rightarrow}\left[\mathrm{C}_{\alpha}, \mathrm{C}_{\alpha}\right] \stackrel{c^{*}}{\rightarrow} \pi_{0,0} \mathrm{C}_{\alpha} \rightarrow 0
$$

then supplies an element $\psi \in \pi_{s+1, w} \mathrm{C}_{\alpha}$ with $\psi \circ d=\mu \circ\left(\mathrm{C}_{\alpha} \wedge c\right)-\mathrm{id}_{\mathrm{C}_{\alpha}}$. The short exact sequence

$$
0 \rightarrow \pi_{2 s+2,2 w} \mathrm{C}_{\alpha} \stackrel{d^{*}}{\rightarrow}\left[\Sigma^{s+1, w} \mathrm{C}_{\alpha}, \mathrm{C}_{\alpha}\right] \stackrel{c^{*}}{\rightarrow} \pi_{s+1, w} \mathrm{C}_{\alpha} \rightarrow 0
$$

provides $\theta: \Sigma^{s, w} \mathrm{C}_{\alpha} \rightarrow \mathrm{C}_{\alpha}$ with $\theta \circ \Sigma^{s+1, w} d=\psi$. It follows that the left unital pairing $\mu-\theta \circ\left(d \wedge \mathrm{C}_{\alpha}\right)$ is also right unital, because

$\left(\mu-\theta \circ\left(d \wedge \mathrm{C}_{\alpha}\right)\right) \circ\left(\mathrm{C}_{\alpha} \wedge c\right)=\mu \circ\left(\mathrm{C}_{\alpha} \wedge c\right)-\theta \circ(d \wedge c)=\mu \circ\left(\mathrm{C}_{\alpha} \wedge c\right)-\theta \circ \Sigma^{s+1, w} c \circ d=\mathrm{id}_{\mathrm{C}_{\alpha}}$.

Hence if a left unital pairing on $\mathrm{C}_{\alpha}$ exists, a unital pairing exists as well. In the following, "multiplication" stands for "unital pairing".

Lemma 5.2. Let $\alpha: \Sigma^{s, w} \mathbf{1} \rightarrow \mathbf{1}$ be an endomorphism. Then $\alpha^{2} \cdot \operatorname{id}_{\mathrm{C}_{\alpha}}=0$.

Proof. The homotopy cofiber sequence defining $\mathrm{C}_{\alpha}$ induces a short exact sequence

$$
0 \rightarrow\left[\Sigma^{s+1, w} \mathbf{1}, \mathrm{C}_{\alpha}\right] / \alpha \stackrel{d^{*}}{\longrightarrow}\left[\mathrm{C}_{\alpha}, \mathrm{C}_{\alpha}\right] \stackrel{c^{*}}{\rightarrow}{ }_{\alpha}\left[\mathbf{1}, \mathrm{C}_{\alpha}\right]=\left[\mathbf{1}, \mathrm{C}_{\alpha}\right]=[\mathbf{1}, \mathbf{1}] / \alpha \rightarrow 0
$$

for which Proposition 3.2 implies the identification of the outer terms. In particular, $c^{*}\left(\operatorname{id}_{\mathrm{C}_{\alpha}}\right)$ is annihilated by $\alpha$. Hence $\alpha \cdot \operatorname{id}_{\mathrm{C}_{\alpha}}=d^{*}(x)$ for some $x \in\left[\Sigma^{s+1, w} \mathbf{1}, \mathrm{C}_{\alpha}\right] / \alpha$. Thus $\alpha \cdot x=0$, which proves the equality $\alpha^{2} \cdot \operatorname{id}_{\mathrm{C}_{\alpha}}=\alpha \cdot d^{*}(x)=d^{*}(\alpha \cdot x)=0$. 
As a consequence, there exists a pairing

$$
\mathrm{C}_{\alpha} \wedge \mathrm{C}_{\alpha^{2}} \rightarrow \mathrm{C}_{\alpha}
$$

which in the classical case of the topological sphere spectrum and $\alpha=2$ was described by Oka in [16]. For example, using $\mathrm{h}^{2}=2 \mathrm{~h}$, there results a pairing

$$
\mathrm{C}_{\mathrm{h}} \wedge \mathrm{C}_{2 \mathrm{~h}} \rightarrow \mathrm{C}_{\mathrm{h}}
$$

which realizes to Oka's pairing for subfields of the complex numbers. The specific role that squares play will be clarified by the following statement lifting a theorem of Brayton Gray [7, Theorem 10].

Theorem 5.3. Let $\alpha: \Sigma^{s, w} \mathbf{1} \rightarrow \mathbf{1}$ be any endomorphism. Then $\mathrm{C}_{\alpha^{2}}$ admits a multiplication.

Proof. Lemma 5.1 implies it suffices to show that $\alpha^{2} \cdot \mathrm{id}_{\mathrm{C}_{\alpha^{2}}}=0$. In order to prove this, consider the following general construction for $\alpha: \mathrm{D} \rightarrow \mathrm{E}$, modeled on [22], and observing that the $\varepsilon$-graded ring structure on $\pi_{*+(\star)} \mathbf{1}$ may be equally defined via composition or via smash product. Let $R(\alpha)$ be the set of all maps $A: \Sigma^{1,0} \mathrm{D} \wedge \mathrm{D} \rightarrow \mathrm{E} \wedge \mathrm{E}$ such the diagram

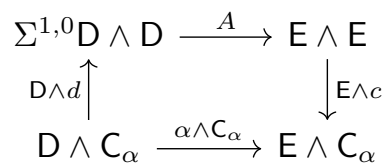

commutes. If $R(\alpha)$ contains an element of the form $\beta \wedge \alpha$, where $\beta: \Sigma^{1,0} \mathrm{D} \rightarrow \mathrm{E}$, then $\alpha \wedge \mathrm{C}_{\alpha}=\alpha \cdot \mathrm{id}_{\mathrm{C}_{\alpha}}$ is the zero map. Several natural commutative diagrams show that if $\alpha: \mathrm{D} \rightarrow \mathrm{E}$ and $\beta: \mathrm{F} \rightarrow \mathrm{G}$ are two maps, then for every $A \in R(\alpha)$, the element

$\Sigma^{1,0} \mathrm{D} \wedge \mathrm{F} \wedge \mathrm{D} \wedge \mathrm{F} \stackrel{\text { twist }}{\longrightarrow} \Sigma^{1,0} \mathrm{D} \wedge \mathrm{D} \wedge \mathrm{F} \wedge \mathrm{F} \stackrel{A \wedge \beta \wedge \beta}{\longrightarrow} \mathrm{E} \wedge \mathrm{E} \wedge \mathrm{G} \wedge \mathrm{G} \stackrel{\text { twist }}{\longrightarrow} \mathrm{E} \wedge \mathrm{G} \wedge \mathrm{E} \wedge \mathrm{G}$ lies in $R(\alpha \wedge \beta)$. In particular, for $\alpha \in \pi_{s, w} \mathbf{1}$ and $\beta \in \pi_{t, x} \mathbf{1}$, the inclusion $(-1)^{(s-w)(t-x)} \varepsilon^{w x} R(\alpha) \beta^{2} \subset R(\alpha \wedge \beta)$ holds, using [4, Equation (2.4)]. In the special case $\alpha=\beta$, one obtains the inclusion $(-1)^{(s-w)^{2}} \varepsilon^{w^{2}} R(\alpha) \alpha^{2} \subset R(\alpha \wedge \alpha)$. If $R(\alpha)$ is not empty, this implies that $\alpha^{2} \wedge \mathrm{C}_{\alpha^{2}}$ is zero. It remains to see that $R(\alpha)$ is not empty for $\alpha \in \pi_{s, w}$. Since $\alpha \wedge C_{\alpha} \circ \Sigma^{s, w} c=c \circ \Sigma^{s, w} \alpha=0$, there exists $\gamma \in \pi_{2 s+1,2 w} \mathrm{C}_{\alpha}$ with $\gamma \circ \Sigma^{s, w} d=\alpha \wedge \mathrm{C}_{\alpha}$. If $d \circ \gamma=0$, then $R(\alpha)$ contains a lift of $\gamma$ along $c$. If $d \circ \gamma \neq 0$, then the equation

$$
d \circ \gamma \circ \Sigma^{s, w} d=d \circ \alpha \wedge C_{\alpha}=\Sigma^{s+1, w} \alpha \circ \Sigma^{s, w} d=0
$$

supplies $\phi \in \pi_{0,0} \mathbf{1}$ with $\phi \circ \alpha=d \circ \gamma$. Since $\alpha \circ \phi \circ \alpha=0$, there exists $\psi \in \pi_{s+1, w} \mathrm{C}_{\alpha}$ with $d \circ \psi \circ \alpha=\phi \circ \alpha$ It follows that $(\gamma-\psi \circ \alpha) \circ d=\gamma \circ d=\alpha \wedge \mathrm{C}_{\alpha}$ and $d \circ(\gamma-\psi \circ \alpha)=0$. A lift of $\gamma-\psi \circ \alpha \in \pi_{2 s+1,2 w} \mathrm{C}_{\alpha}$ along $c$ provides an element in $R(\alpha)$, which concludes the proof.

Now for some negative results regarding multiplications on motivic Moore spectra. Recall that $\pi_{0,0} \mathbf{1}=G W$ identifies with the Grothendieck-Witt ring of quadratic forms by Theorem 2.1, which comes equipped with a dimension ring homomorphism dim: $\mathbf{G W} \rightarrow \mathbb{Z}$.

Theorem 5.4. Let $\alpha: \mathbf{1} \rightarrow \mathbf{1}$ be an endomorphism with $\operatorname{dim}(\alpha) \equiv 2(4)$. Then $\mathrm{C}_{\alpha}$ does not admit a multiplication. 
Proof. This result follows by complex realization from [16] for subfields of the complex numbers. In any case, consider motivic cohomology $h^{*, *}$ with coefficients in $\mathbb{F}_{2}$. Since multiplication with $\alpha$ induces multiplication with $\operatorname{dim}(\alpha)$ on motivic cohomology, there results a split short exact sequence

$$
0 \rightarrow h^{*, *} \rightarrow h^{*, *}\left(\mathrm{C}_{\alpha}\right) \rightarrow h^{*+1, *} \rightarrow 0
$$

on motivic cohomology. Pick basis elements $x_{0} \in h^{0,0}\left(\mathrm{C}_{\alpha}\right), x_{1} \in h^{1,0}\left(\mathrm{C}_{\alpha}\right)$, and note that $\mathrm{Sq}^{1}\left(x_{0}\right)=x_{1}$ since $\operatorname{dim}(\alpha) \equiv 2(4)$. The split short exact sequence

$$
0 \rightarrow h^{*, *}\left(\mathrm{C}_{\alpha}\right) \rightarrow h^{*, *}\left(\mathrm{C}_{\alpha} \wedge \mathrm{C}_{\alpha}\right) \rightarrow h^{*+1, *}\left(\mathrm{C}_{\alpha}\right) \rightarrow 0
$$

induced by multiplication with $\alpha$ on $\mathrm{C}_{\alpha}$ then supplies $h^{*, *}\left(\mathrm{C}_{\alpha} \wedge \mathrm{C}_{\alpha}\right)$ with basis elements $x_{0} \otimes y_{0}, x_{1} \otimes y_{0}, x_{0} \otimes y_{1}, x_{1} \otimes y_{1}$. Here $y_{0} \in h^{0,0}\left(\mathrm{C}_{\alpha}\right)$ and $y_{1} \in h^{1,0}\left(\mathrm{C}_{\alpha}\right)$ are basis elements in the other factor of the smash product $C_{\alpha} \wedge C_{\alpha}$. A Künneth theorem supplies the basis elements for the motivic cohomology of the smash product. The Cartan formula

$$
\mathrm{Sq}^{2}\left(x_{0} \otimes y_{0}\right)=\mathrm{Sq}^{2}\left(x_{0}\right) \otimes y_{0}+x_{0} \otimes \mathrm{Sq}^{2}\left(y_{0}\right)+\tau \mathrm{Sq}^{1}\left(x_{0}\right) \otimes \mathrm{Sq}^{1}\left(y_{0}\right)=\tau x_{1} \otimes y_{1}
$$

from [23, Proposition 9.7] then shows that $\mathrm{Sq}^{2}$ acts nontrivially on $h^{* * *}\left(\mathrm{C}_{\alpha} \wedge \mathrm{C}_{\alpha}\right)$. If $\alpha \cdot \operatorname{id}_{\mathrm{C}_{\alpha}}=0$, then $\mathrm{C}_{\alpha} \wedge \mathrm{C}_{\alpha}=\mathrm{C}_{\alpha} \vee \Sigma^{1,0} \mathrm{C}_{\alpha}$, and $h^{*, *}\left(\mathrm{C}_{\alpha} \wedge \mathrm{C}_{\alpha}\right)$ splits accordingly as a module over the motivic Steenrod algebra, implying $\mathrm{Sq}^{2}\left(x_{0} \otimes y_{0}\right)=0$. The result follows.

Lemma 5.5. The motivic Moore spectrum $\mathrm{C}_{\eta^{\ell}}$ admits a multiplication if and only if $\ell>1$.

Proof. Let us prove first that $C_{\eta}$ does not admit a multiplication. Again an argument via complex realization works for subfields of the complex numbers. However, one may refer to Example 4.3 and Proposition 4.2 which imply the existence of an element $\tilde{\nu} \in \pi_{3,2} \mathrm{C}_{\eta}$ such that $\tilde{\nu} \cdot \eta \neq 0$. In particular, $\eta \cdot \operatorname{id}_{\mathrm{C}_{\eta}} \neq 0$.

Consider now $\ell>1$. The element $\eta^{\ell} \cdot \mathrm{id}_{\mathrm{C}_{\eta^{\ell}}}$ lies inside the group which sits in the middle of the short exact sequence

$$
0 \rightarrow\left[\Sigma^{2 \ell+1,2 \ell} \mathbf{1}, \mathrm{C}_{\eta^{\ell}}\right] / \eta^{\ell} \stackrel{d^{*}}{\rightarrow}\left[\Sigma^{\ell, \ell} \mathrm{C}_{\eta^{\ell}}, \mathrm{C}_{\eta^{\ell}}\right] \stackrel{c^{*}}{\rightarrow} \eta_{\eta^{\ell}}\left[\Sigma^{\ell, \ell} \mathbf{1}, \mathrm{C}_{\eta^{\ell}}\right]=\left[\Sigma^{\ell, \ell} \mathbf{1}, \mathbf{1}\right] / \eta^{\ell} \rightarrow 0
$$

whose final term is zero, since $\eta^{\ell}: \pi_{0,0} \mathbf{1} \rightarrow \pi_{\ell, \ell} \mathbf{1}$ is surjective. The initial term is computed by Proposition 3.2. More precisely, the group $\pi_{2 \ell+1,2 \ell} \mathrm{C}_{\eta^{\ell}}$ sits inside the short exact sequence

$$
0 \rightarrow \pi_{2 \ell+1,2 \ell} \mathbf{1} / \eta^{\ell} \pi_{\ell+1, \ell} \mathbf{1} \stackrel{c_{*}}{\rightarrow} \pi_{2 \ell+1,2 \ell} \mathrm{C}_{\eta^{\ell}} \stackrel{d_{*}}{\longrightarrow} \eta^{\ell} \pi_{\ell, \ell} \mathbf{1} \rightarrow 0
$$

where ${ }_{\eta^{\ell}} \pi_{\ell, \ell} \mathbf{1}=0$ since $\eta^{\ell}: \pi_{\ell, \ell} \mathbf{1} \rightarrow \pi_{2 \ell, 2 \ell} \mathbf{1}$ is an isomorphism for $\ell \geq 1$. The group $\pi_{2 \ell+1,2 \ell} \mathbf{1}$ vanishes by Theorem 2.2 for $\ell>1$, whence so does $\pi_{2 \ell+1,2 \ell} C_{\eta^{\ell}}$, and thus also $\left[\Sigma^{\ell, \ell} \mathrm{C}_{\eta^{\ell}}, \mathrm{C}_{\eta^{\ell}}\right]$.

The existence of a multiplication may depend on the base field in general. Example 4.4 and Lemma 5.1 show that the motivic Moore spectrum $\mathrm{C}_{2+11 \mathrm{~h}}$ admits no multiplication over a formally real field $F$. Base change to $F(\sqrt{-1})$ produces the motivic Moore spectrum $\mathrm{C}_{2+11 \mathrm{~h}}=\mathrm{C}_{24}$, which admits a multiplication by lifting from topology and quoting [16]. Plenty of similar examples may be constructed. A systematic study on these matters remains a project for the future. Another project for the future is to enumerate possible multiplications, as well as investigate their qualitative properties like associativity and commutativity. Already the 
enumeration can be challenging. For example, the group $\left[C_{h^{2}} \wedge C_{h^{2}}, C_{h^{2}}\right]$ receives a nontrivial map from $\left[\Sigma^{1,0} C_{h^{2}}, C_{h^{2}}\right]$, which is injective on the contribution from the $\mathrm{h}^{2}$-torsion in $\pi_{0,0} \mathbf{1}$ (see Proposition 3.2). The latter coincides with the fundamental ideal in the Grothendieck-Witt ring, as the following statement implies.

Lemma 5.6. Let $\alpha \in \mathbf{G W}(F)$ be an element with $\operatorname{dim}(\alpha) \neq 0$. Then

$$
{ }_{\alpha \mathrm{h}} \mathbf{G W}(F)={ }_{\operatorname{dim}(\alpha) \mathrm{h}} \mathbf{G W}(F)={ }_{\mathrm{h}} \mathbf{G W}(F)=\mathbf{I}(F) .
$$

Proof. Recall that $\mathrm{h}=1-\epsilon=\langle 1,-1\rangle$ is a form of dimension two with the property that the subgroup of $\mathbf{G W}(F)$ generated by $\mathrm{h}$ coincides with the ideal of $\mathbf{G W}(F)$ generated by $h$. The latter follows from the similarity

$$
\langle u\rangle \cdot\langle 1,-1\rangle=\langle u,-u\rangle \sim\langle 1,-1\rangle
$$

of quadratic forms, where $u \in F^{\times}$. Hence for every $\alpha \in \mathbf{G W}(F)$, the equation $\alpha \cdot \mathrm{h}=\operatorname{dim}(\alpha) \cdot \mathrm{h}$ follows, giving the first equation. Also the other equations follow for $\operatorname{dim}(\alpha) \neq 0$, since $\beta \in \mathbf{G W}(F)$ then satisfies $\beta \cdot(\alpha \cdot \mathrm{h})=0$ if and only if $\operatorname{dim}(\beta)=0$. Here recall the short exact sequence

$$
0 \rightarrow \mathbf{I}(F) \rightarrow \mathbf{G W}(F) \stackrel{\operatorname{dim}}{\longrightarrow} \mathbb{Z} \rightarrow 0
$$

defining the fundamental ideal in $\mathbf{G W}(F)$.

Prompted by a recent request, this section concludes with a specific example. Let $n>0$ be a natural number, and let $n_{\varepsilon}=\sum_{k=0}^{n-1}\left\langle(-1)^{k}\right\rangle=\langle 1,-1,1 \ldots, \pm 1\rangle \in \mathbf{G W}$, a quadratic form of dimension $n$. It turns out that the motivic Moore spectrum $\mathrm{C}_{n_{\varepsilon}}$ admits a multiplication precisely if the topological Moore spectrum $\mathbb{S} / n$ does. Before proving this, set $e: \mathrm{C}_{\alpha} \stackrel{d}{\rightarrow} \Sigma^{s+1, w} \mathbf{1} \stackrel{\Sigma^{s+1, w} c}{\longrightarrow} \Sigma^{s+1, w} \mathrm{C}_{\alpha}$ for $\alpha \in \pi_{s, w} \mathbf{1}$. Following [16], a multiplication $\mu: \mathrm{C}_{\alpha} \wedge \mathrm{C}_{\alpha} \rightarrow \mathrm{C}_{\alpha}$ is called regular if the equality $e \circ \mu=\left(\Sigma^{s+1, w} \mu\right) \circ\left(e \wedge \mathrm{C}_{\alpha}+\mathrm{C}_{\alpha} \wedge e\right): \mathrm{C}_{\alpha} \wedge \mathrm{C}_{\alpha} \rightarrow \Sigma^{s+1, w} \mathrm{C}_{\alpha}$ holds.

Lemma 5.7. There exists a multiplication on $\mathrm{C}_{n_{\varepsilon}}$ if and only if $n \not \equiv 2(4)$.

Proof. Theorem 5.4 says that $C_{n_{\varepsilon}}$ does not admit a multiplication if $n \equiv 2(4)$. Suppose first that $n=2 m+1$ is an odd natural number. Any element $\alpha \in \mathbf{G W}$ with $n_{\varepsilon} \alpha=0$ then satisfies $\operatorname{dim}(\alpha)=0$, and hence lies in the fundamental ideal. Since $n_{\varepsilon}=1+m \mathrm{~h}$ and the element $\mathrm{h} \alpha$ is hyperbolic of dimension zero, one concludes $\alpha=0$. Proposition 3.2 then provides an isomorphism $\pi_{1,0} \mathrm{C}_{n_{\varepsilon}} \cong \pi_{1,0} \mathbf{1} / n_{\varepsilon} \pi_{1,0} \mathbf{1}$. Lemma 2.6 implies that the map $\pi_{1,0} \mathbf{1} \stackrel{n_{\varepsilon}}{\longrightarrow} \pi_{1,0} \mathbf{1}$ is surjective if $n \not \equiv 0(3)$. Hence if $n$ is odd and not divisible by three, $\pi_{1,0} C_{n_{\varepsilon}}=0$. The short exact sequence (5.1) then reduces to $\left[\mathrm{C}_{n_{\varepsilon}}, \mathrm{C}_{n_{\varepsilon}}\right] \cong \pi_{0,0} \mathrm{C}_{n_{\varepsilon}}$. In particular, the identity on $\mathrm{C}_{n_{\varepsilon}}$ is annihilated by $n_{\varepsilon}$, which provides the existence of a multiplication by Lemma 5.1. If $n$ is odd and divisible by three, $\pi_{1,0} \mathrm{C}_{n_{\varepsilon}} \cong \mathbf{K}_{2}^{\mathrm{M}} / 3$. In order to conclude for such odd numbers as well, it suffices to prove that the short exact sequence

$$
0 \rightarrow \pi_{1,0} \mathrm{C}_{n_{\varepsilon}} \cong \mathbf{K}_{2}^{\mathrm{M}} / 3 \rightarrow\left[\mathrm{C}_{n_{\varepsilon}}, \mathrm{C}_{n_{\varepsilon}}\right] \rightarrow \pi_{0,0} \mathrm{C}_{n_{\varepsilon}} \rightarrow 0
$$

splits. Because $n_{\varepsilon}=1+m \cdot \mathrm{h}$ acts as the identity on the Witt group, $\pi_{0,0} \mathrm{C}_{n_{\varepsilon}} \cong \mathbb{Z} / n$ for any field, generated by $h$. Hence if the sequence (5.2) splits over prime fields, it does so over any field, reducing the task to $F=\mathbb{Q}$. In this case, 13, Theorem 11.6] supplies an isomorphism $\mathbf{K}_{2}^{\mathrm{M}} / 3(\mathbb{Q}) \cong \underset{p \text { prime, } p \equiv 1(3)}{\bigoplus} \mathbb{Z} / 3$ whose contribution at 
the prime $p \equiv 1(3)$ is induced by the localization sequence

$$
\operatorname{Spec}\left(\mathbb{F}_{p}\right) \hookrightarrow \operatorname{Spec}\left(\mathbb{Z}_{(p)}\right) \hookleftarrow \operatorname{Spec}(\mathbb{Q})
$$

Since both $\mathrm{h}$ and the element $n_{\varepsilon}$ are defined over $\operatorname{Spec}(\mathbb{Z})$, it follows that the sequence (5.2) splits.

Suppose now that $n=2^{r} m$ for some odd natural number $m$, with $r \geq 2$. Then $n_{\varepsilon}=m \mathrm{~h}^{r}$. By Theorem [5.3. $\mathrm{C}_{\mathrm{h}^{2}}$ admits a multiplication $\mu$. If $\mu$ is not regular, the difference $x=e \circ \mu-\mu \circ\left(e \wedge \mathrm{C}_{\mathrm{h}^{2}}+\mathrm{C}_{\mathrm{h}^{2}} \wedge e\right)$ lifts to produce a unique element $y \in \pi_{1,0} \mathrm{C}_{\mathrm{h}^{2}}$ such that $y \circ(d \wedge d)=x$. The latter equation shows that $x$ is an element of order two, because $d \wedge d=-d \wedge d$, hence so is $y$. The vanishing $0=d \circ y \in \pi_{0,0} \mathrm{C}_{\mathrm{h}^{2}}$ implies that there exists $z \in \pi_{1,0} \mathbf{1}$ with $c \circ z=y$. The image of $\pi_{1,0} \mathbf{1}$ under composition with $c$ is isomorphic, by Theorem 2.5 to $\mathbf{K}_{2}^{\mathrm{M}} / 4 \oplus \mathbf{k}_{1}^{\mathrm{M}} \oplus \mathbf{k}_{0}^{\mathrm{M}}$. The map $\lambda: \mathrm{C}_{\mathrm{h}^{2}} \rightarrow \mathrm{C}_{\mathrm{h}^{4}}$ induced by multiplication with $\mathrm{h}^{2}$ on the bottom cell does not necessarily induce the zero homomorphism on $\pi_{1,0}$. More specifically, it induces multiplication by 4 as a homomorphism

$$
\mathbf{K}_{2}^{\mathrm{M}} / 4 \oplus \mathbf{k}_{1}^{\mathrm{M}} \oplus \mathbf{k}_{0}^{\mathrm{M}} \rightarrow \mathbf{K}_{2}^{\mathrm{M}} / 8 \oplus \mathbf{k}_{1}^{\mathrm{M}} \oplus \mathbf{k}_{0}^{\mathrm{M}}
$$

and in particular sends the 2-torsion element $y$ to zero. The homotopy cofiber sequence

$$
\mathrm{C}_{\mathrm{h}^{2}} \stackrel{\lambda}{\rightarrow} \mathrm{C}_{\mathrm{h}^{2}} \stackrel{\rho}{\rightarrow} \mathrm{C}_{\mathrm{h}^{2}} \stackrel{e}{\rightarrow} \Sigma^{1,0} \mathrm{C}_{\mathrm{h}^{2}}
$$

implies the existence of $w \in \pi_{2,0} \mathrm{C}_{\mathrm{h}^{2}}$ with $e \circ w=y$. Then $\mu-w \circ(d \wedge d)$ is a regular multiplication on $C_{h^{2}}$. Hence a regular multiplication exists on $C_{h^{2}}$.

Inductively, one may lift a regular multiplication on $C_{h}$ e to a regular multiplication on $\mathrm{C}_{\mathrm{h} \ell+1}$ via the homotopy cofiber sequence

$$
\mathrm{C}_{\mathrm{h}} \stackrel{\lambda}{\rightarrow} \mathrm{C}_{\mathrm{h} \ell+1} \stackrel{\rho}{\rightarrow} \mathrm{C}_{\mathrm{h}^{\ell}} \stackrel{\delta}{\rightarrow} \mathrm{C}_{\mathrm{h}} .
$$

Here $\lambda$ and $\rho$ are induced by suitable multiplications with $\mathrm{h}^{\ell}$ on the bottom cell and $\mathrm{h}$ on the top cell, respectively, and $\delta$ is the composition $\mathrm{C}_{\mathrm{h}} \stackrel{d}{\rightarrow} \Sigma^{1,0} \mathbf{1} \stackrel{\Sigma^{1,0} c}{\longrightarrow} \mathrm{C}_{\mathrm{h}}$. The homotopy cofiber sequence

$$
\mathrm{C}_{\mathrm{h}} \stackrel{\lambda}{\rightarrow} \mathrm{C}_{m} \stackrel{\rho}{\rightarrow} \mathrm{C}_{m \mathrm{~h}^{r}} \stackrel{\delta}{\rightarrow} \mathrm{C}_{\mathrm{h}^{r}}
$$

allows to lift a regular multiplication $\mu$ on $\mathrm{C}_{\mathrm{h}^{r}}$ to a regular multiplication on $\mathrm{C}_{n_{\varepsilon}}$. Over quadratically closed fields, $\delta=0$ (as in topology, see [16, Lemma 5]), but $\delta \neq 0$ for formally real fields. Nevertheless the equality $\delta \circ \mu \circ(\rho \wedge \rho)=0$ always holds. This computation follows from the fact that $\mu$ is regular and two other inputs. One input is the result from topology that $m$ is zero on $\mathbb{S} / m$, and hence also on its image $C_{m}$ in the motivic stable homotopy category. In order to apply the regularity, one uses that the composition $\mathrm{C}_{\mathrm{h}^{r}} \stackrel{d}{\rightarrow} \Sigma^{1,0} \mathbf{1} \stackrel{\Sigma^{1,0} c}{\longrightarrow} \Sigma^{1,0} \mathrm{C}_{m}$ factors as $\mathrm{C}_{\mathrm{h}^{r}} \stackrel{d}{\rightarrow} \Sigma^{1,0} \mathbf{1} \stackrel{\Sigma^{1,0} c}{\longrightarrow} \Sigma^{1,0} \mathrm{C}_{\mathrm{h}^{r}} \stackrel{\Sigma^{1,0} \phi}{\rightarrow} \Sigma^{1,0} \mathrm{C}_{m}$ for some $\phi: \mathrm{C}_{\mathrm{h}^{r}} \rightarrow \mathrm{C}_{m}$. This provides a regular multiplication on $\mathrm{C}_{n_{\varepsilon}}$ for $n \not \equiv 2(4)$, which concludes the proof.

\section{Slices of Motivic Moore SPeCtra}

Instead of considering slices for general motivic Moore spectra, the focus here motivated by arguments regarding the vanishing of higher slice differentials in [21, Section 4] - is on $C_{n \mathrm{~h}}$, where $0<n \in \mathbb{N}$. As explained in the proof of Lemma [5.6, it suffices to consider motivic Moore spectra with respect to $n \mathrm{~h}$ instead of $\alpha \mathrm{h}$ for $\alpha \in \mathbf{G W}(F)$ of $\operatorname{dim}(\alpha) \neq 0$. Since $\mathbf{h}=0: \mathrm{KW} \rightarrow \mathrm{KW}$ as an endomorphism on the 
motivic spectrum representing higher Witt groups, the motivic spectrum $\mathrm{KW}_{n \mathrm{~h}}$ splits as $\mathrm{KW} \vee \Sigma^{1,0} \mathrm{KW}$. The same holds for its (effective or connective) covers, and also for the corresponding slices. In particular, the first slice differential for $\mathrm{KW}_{n \mathrm{~h}}$ splits. For reference purposes, the explicit form is as follows.

Theorem 6.1. Let $0<n \in \mathbb{N}$. The restriction of the slice $\mathrm{d}^{1}$-differential to the summand $\Sigma^{q+j, q} \mathbf{M} \mathbb{Z} / 2$ of $\mathbf{s}_{q}\left(\mathrm{KW}_{n \mathrm{~h}}\right)$ is given by

$$
\mathrm{d}^{1}\left(\mathrm{KW}_{n \mathrm{~h}}\right)(q, j)= \begin{cases}\left(\mathrm{Sq}^{3} \mathrm{Sq}^{1}, 0, \mathrm{Sq}^{2}\right) & j \equiv 0,1 \bmod 4 \\ \left(\mathrm{Sq}^{3} \mathrm{Sq}^{1}, 0, \mathrm{Sq}^{2}+\rho \mathrm{Sq}^{1}, 0, \tau\right) & j \equiv 2,3 \bmod 4\end{cases}
$$

Here the ith component of the map $\mathrm{d}^{1}\left(\mathrm{KW}_{n \mathrm{~h}}\right)(q, j)$ of motivic spectra is a map $\Sigma^{q+j, q} \mathbf{M} \mathbb{Z} / 2 \rightarrow \Sigma^{q+j+i, q+1} \mathbf{M} \mathbb{Z} / 2$.

Proof. This follows from the determination of the first slice differential for $\mathrm{KW}$ from [19], and the aforementioned splitting $\mathrm{KW}_{n \mathrm{~h}} \simeq \mathrm{KW} \vee \Sigma^{1,0} \mathrm{KW}$.

Here and in the following, the notation regarding the motivic Steenrod algebra is standard; for example, $\rho$ is the class of -1 in $h^{1,1}$, and $\mathrm{Q}_{1}=\mathrm{Sq}^{2} \mathrm{Sq}^{1}+\mathrm{Sq}^{1} \mathrm{Sq}^{2}$. The slice computation is slightly more complicated for the motivic spectrum $K Q$ representing hermitian $K$-theory. For comparison purposes, the case of $\mathrm{kq}$, the very effective cover of $K Q$ 1], 2, is more convenient. Set $\mathrm{kq}_{n \mathrm{~h}}:=\mathrm{kq} \wedge \mathrm{C}_{n \mathrm{~h}}$, and similarly $\mathrm{cKW}_{n \mathrm{~h}}:=\mathrm{cKW} \wedge \mathrm{C}_{n \mathrm{~h}}=\mathrm{cKW} \vee \Sigma^{1,0} \mathrm{cKW}$. Here $\mathrm{cKW}:=\mathrm{KW} \geq 0 \cong \mathrm{kq}\left[\eta^{-1}\right]$ is the connective cover of $K W$, not its very effective cover (which might deserve the notation $\mathrm{kw}$ ). The element $\eta$ acts still invertibly on cKW.

The final set of notation concerns long exact sequences of motivic cohomology groups induced by change of coefficients in cyclic groups. Given natural numbers $m, n$, the inclusion $\mathbb{Z} / m \hookrightarrow \mathbb{Z} / m n$ induces a homomorphism on motivic cohomology denoted inc $m n$, and the projection $\mathbb{Z} / m n \rightarrow \mathbb{Z} / n$ induces a homomorphism on motivic cohomology denoted $\mathrm{pr}_{n}^{m n}$. The short exact sequence

$$
0 \rightarrow \mathbb{Z} / m \rightarrow \mathbb{Z} / m n \rightarrow \mathbb{Z} / n \rightarrow 0
$$

induces a Bockstein or boundary homomorphism $\partial_{m}^{n}$.

Theorem 6.2. Let $0<n, 0 \leq q$. The slices of $\mathrm{kq}_{n \mathrm{~h}}$ are given as follows:

$$
\begin{aligned}
\mathrm{s}_{2 q}\left(\mathrm{kq}_{n \mathrm{~h}}\right) & =\Sigma^{4 q, 2 q} \mathbf{M} \mathbb{Z} / 2 n \vee \bigvee_{j=0}^{2 q-1} \Sigma^{2 q+j, 2 q} \mathbf{M} \mathbb{Z} / 2 \\
\mathrm{~s}_{2 q+1}\left(\mathrm{kq}_{n \mathrm{~h}}\right) & =\mathrm{s}_{2 q+1}(\mathrm{kq}) \vee \Sigma^{1,0} \mathrm{~s}_{2 q+1}(\mathrm{kq})=\bigvee_{j=0}^{2 q+1} \Sigma^{2 q+1+j, 2 q+1} \mathbf{M} \mathbb{Z} / 2
\end{aligned}
$$

The canonical map $\mathrm{kq}_{n \mathrm{~h}} \rightarrow \mathrm{cKW}_{n \mathrm{~h}}$ induces the following map on summands of slices:

$$
\begin{aligned}
& \Sigma^{4 q, 2 q} \mathbf{M} \mathbb{Z} / 2 n \stackrel{\left(\partial_{2}^{2 n}, \mathrm{pr}_{2}^{2 n}\right)}{\longrightarrow} \Sigma^{4 q+1,2 q} \mathbf{M} \mathbb{Z} / 2 \vee \Sigma^{4 q, 2 q} \mathbf{M} \mathbb{Z} / 2 \\
& \Sigma^{q+j, q} \mathbf{M} \mathbb{Z} / 2 \stackrel{(1)}{\longrightarrow} \Sigma^{q+j, q} \mathbf{M} \mathbb{Z} / 2 \quad \text { if } j \equiv 0(2) \text { or } n \equiv 0(2) \\
& \Sigma^{q+j, q} \mathbf{M} \mathbb{Z} / 2 \stackrel{\left(\mathrm{Sq}^{1}, 1\right)}{\longrightarrow} \Sigma^{q+j+1, q} \mathbf{M} \mathbb{Z} / 2 \vee \Sigma^{q+j, q} \mathbf{M} \mathbb{Z} / 2 \quad \text { if } j \equiv 1(2) \text { and } n \equiv 1(2)
\end{aligned}
$$


The restriction of the slice $\mathrm{d}^{1}$-differential for $\mathrm{kq}_{n \mathrm{~h}}$ to the summands $\Sigma^{q+j, q} \mathbf{M} \mathbb{Z} / 2$ or $\Sigma^{4 q, 2 q} \mathbf{M} \mathbb{Z} / 2$ of $\mathbf{s}_{q}\left(\mathrm{kq}_{n \mathrm{~h}}\right)$ for $n$ even is given by

$$
\begin{array}{r}
\mathrm{d}^{1}\left(\mathrm{kq}_{n \mathrm{~h}}\right)(q, j)= \begin{cases}\left(\mathrm{Sq}^{3} \mathrm{Sq}^{1}, 0, \mathrm{Sq}^{2}\right) & q-1>j \equiv 0,1 \bmod 4 \\
\left(\mathrm{Sq}^{3} \mathrm{Sq}^{1}, 0, \mathrm{Sq}^{2}+\rho \mathrm{Sq}^{1}, 0, \tau\right) & q-1>j \equiv 2,3 \bmod 4\end{cases} \\
\mathrm{d}^{1}\left(\mathrm{kq}_{n \mathrm{~h}}\right)(q, q-1)= \begin{cases}\left(\partial_{2 n}^{2} \mathrm{Sq}^{2} \mathrm{Sq}^{1}, 0, \mathrm{Sq}^{2}\right) & q-1 \equiv 0 \bmod 4 \\
\left(\mathrm{Sq}^{3} \mathrm{Sq}^{1}, 0, \mathrm{Sq}^{2}\right) & q-1 \equiv 1 \bmod 4 \\
\left(\partial_{2 n}^{2} \mathrm{Sq}^{2} \mathrm{Sq}^{1}, 0, \mathrm{Sq}^{2}+\rho \mathrm{Sq}^{1}, 0, \tau\right) & q-1 \equiv 2 \bmod 4 \\
\left(\mathrm{Sq}^{3} \mathrm{Sq}^{1}, 0, \mathrm{Sq}^{2}+\rho \mathrm{Sq}^{1}, 0, \tau\right) & q-1 \equiv 3 \bmod 4 .\end{cases} \\
\mathrm{d}^{1}\left(\mathrm{kq}_{n \mathrm{~h}}\right)(q, q)= \begin{cases}\left(\mathrm{Sq}^{2} \partial_{2}^{2 n}, \mathrm{Sq}^{2} \mathrm{pr}_{2}^{2 n}\right) & q \equiv 0 \bmod 4 \\
\left(\mathrm{inc}_{2 n}^{2} \mathrm{Sq}^{2} \mathrm{Sq}^{1}, \mathrm{Sq}^{2}\right) & q \equiv 1 \bmod 4 \\
\left(\mathrm{Sq}^{2} \partial_{2}^{2 n}, \mathrm{Sq}^{2} \mathrm{pr}_{2}^{2 n}, \tau \partial_{2}^{2 n}, \tau \mathrm{pr}_{2}^{2 n}\right) & q \equiv 2 \bmod 4 \\
\left(\mathrm{inc}_{2 n}^{2} \mathrm{Sq}^{2} \mathrm{Sq}^{1}, \mathrm{Sq}^{2}+\rho \mathrm{Sq}^{1}, 0, \tau\right) & q \equiv 3 \bmod 4 .\end{cases}
\end{array}
$$

The restriction of the slice $\mathrm{d}^{1}$-differential for $\mathrm{kq}_{n \mathrm{~h}}$ to the summands $\Sigma^{q+j, q} \mathbf{M} \mathbb{Z} / 2$ or $\Sigma^{4 q, 2 q} \mathbf{M} \mathbb{Z} / 2 n$ of $\mathbf{s}_{q}\left(\mathrm{kq}_{n \mathrm{~h}}\right)$ for $n$ odd is given by

$$
\begin{array}{r}
\mathrm{d}^{1}\left(\mathrm{kq}_{n \mathrm{~h}}\right)(q, j)= \begin{cases}\left(\mathrm{Sq}^{3} \mathrm{Sq}^{1}, 0, \mathrm{Sq}^{2}\right) & q-1>j \equiv 0 \bmod 4 \\
\left(\mathrm{Sq}^{3} \mathrm{Sq}^{1}, \mathrm{Q}_{1}, \mathrm{Sq}^{2}, \tau \mathrm{Sq}^{1}\right) & q-1>j \equiv 1 \bmod 4 \\
\left(\mathrm{Sq}^{3} \mathrm{Sq}^{1}, 0, \mathrm{Sq}^{2}+\rho \mathrm{Sq}^{1}, 0, \tau\right) & q-1>j \equiv 2 \bmod 4 \\
\left(\mathrm{Sq}^{3} \mathrm{Sq}^{1}, \mathrm{Q}_{1}, \mathrm{Sq}^{2}+\rho \mathrm{Sq}^{1}, \tau \mathrm{Sq}^{1}+\rho, \tau\right) & q-1>j \equiv 3 \bmod 4,\end{cases} \\
\mathrm{d}^{1}\left(\mathrm{kq}_{n \mathrm{~h}}\right)(q, q-1)= \begin{cases}\left(\partial_{2 n}^{2} \mathrm{Sq}^{2} \mathrm{Sq}^{1}, 0, \mathrm{Sq}^{2}\right) & q-1 \equiv 0 \bmod 4 \\
\left(\mathrm{Sq}^{3} \mathrm{Sq}^{1}, \mathrm{Q}_{1}, \mathrm{Sq}^{2}, \tau \mathrm{Sq}^{1}\right) & q-1 \equiv 1 \bmod 4 \\
\left(\partial_{2 n}^{2} \mathrm{Sq}^{2} \mathrm{Sq}^{1}, 0, \mathrm{Sq}^{2}+\rho \mathrm{Sq}^{1}, 0, \tau\right) & q-1 \equiv 2 \bmod 4 \\
\left(\mathrm{Sq}^{3} \mathrm{Sq}^{1}, \mathrm{Q}_{1}, \mathrm{Sq}^{2}+\rho \mathrm{Sq}^{1}, \tau \mathrm{Sq}^{1}+\rho, \tau\right) & q-1 \equiv 3 \bmod 4 .\end{cases} \\
\mathrm{d}^{1}\left(\mathrm{kq}_{n \mathrm{~h}}\right)(q, q)= \begin{cases}\left(\mathrm{Sq}^{2} \partial_{2}^{2 n}, \mathrm{Sq}^{2} \mathrm{pr}_{2}^{2 n}\right) & q \equiv 0 \bmod 4 \\
\left(\mathrm{inc}_{2 n}^{2} \mathrm{Sq}^{2} \mathrm{Sq}^{1}+\partial_{2 n}^{2} \mathrm{Sq}^{2}, \mathrm{Sq}^{2}, \tau \mathrm{Sq}^{1}\right) & q \equiv 1 \bmod 4 \\
\left(\mathrm{Sq}^{2} \partial_{2}^{2 n}, \mathrm{Sq}^{2} \mathrm{pr}_{2}^{2 n}, \tau \partial_{2}^{2 n}, \tau \mathrm{pr}_{2}^{2 n}\right) & q \equiv 2 \bmod 4 \\
\left(\mathrm{inc}_{2 n}^{2} \mathrm{Sq}^{2} \mathrm{Sq}^{1}+\partial_{2 n}^{2} \mathrm{Sq}^{2}, \mathrm{Sq}^{2}+\rho \mathrm{Sq}^{1}, \tau \mathrm{Sq}^{1}+\rho, \tau\right) & q \equiv 3 \bmod 4 .\end{cases}
\end{array}
$$

Proof. The description of the slices follows from the fact that the slice functors are triangulated, and [1, Theorem 3.2]. The effect of the canonical map $\mathrm{kq}_{n \mathrm{~h}} \rightarrow \mathrm{cKW}_{n \mathrm{~h}}$ on slices is readily obtained, except for the occurrence of $\mathrm{Sq}^{1}$. The latter follows from the determination of the first slice differential for $\mathrm{cKW}_{n \mathrm{~h}}=\mathrm{cKW} \vee \Sigma^{1,0} \mathrm{cKW}$, compared with possible first slice differentials for $\mathrm{kq}_{n h}$ compatible with the first slice differential for $\mathrm{kq}$, as described in [1, Theorem 3.5]. Determining the first slice differential for $\mathrm{kq}_{n \mathrm{~h}}$ is then essentially straightforward.

Let $1<n \in \mathbb{N}$, set $\mathrm{kq}_{n}:=\mathrm{kq} \wedge \mathrm{C}_{n}$, and $\mathrm{cKW}_{n}:=\mathrm{cKW} \wedge \mathrm{C}_{n}$. For comparison, consider the form of the first slice differential for $\mathrm{cKW}_{2}$, as obtained in 9, Theorem 4.3, Theorem 4.14]. Despite the abstract isomorphisms $\mathrm{s}_{*} \mathrm{cKW}_{2} \cong \mathrm{s}_{*} \mathrm{cKW}_{\mathrm{h}}$ and $\mathrm{s}_{*} \mathrm{kq}_{2} \cong \mathrm{s}_{*} \mathrm{kq}_{\mathrm{h}}$, the first slice differentials differ. Note that if $n$ is odd, then all slices of $\mathrm{cKW}_{n}$ vanish, although $\mathrm{cKW}_{n}$ itself does not over formally real fields. 
Theorem 6.3. The restriction of the slice $\mathrm{d}^{1}$-differential for $\mathrm{cKW}_{2}$ to the summand $\Sigma^{q+j, q} \mathbf{M} \mathbb{Z} / 2$ of $\mathbf{s}_{q}\left(\mathrm{cKW}_{2}\right)$ is given by

$$
\mathrm{d}^{1}\left(\mathrm{cKW}_{2}\right)(q, j)= \begin{cases}\left(\mathrm{Sq}^{3} \mathrm{Sq}^{1}, 0, \mathrm{Sq}^{2}\right) & j \equiv 0 \bmod 4 \\ \left(\mathrm{Sq}^{3} \mathrm{Sq}^{1}, \mathrm{Q}_{1}, \mathrm{Sq}^{2}, \rho+\tau \mathrm{Sq}^{1}\right) & j \equiv 1 \bmod 4 \\ \left(\mathrm{Sq}^{3} \mathrm{Sq}^{1}, 0, \mathrm{Sq}^{2}+\rho \mathrm{Sq}^{1}, 0, \tau\right) & j \equiv 2 \bmod 4 \\ \left(\mathrm{Sq}^{3} \mathrm{Sq}^{1}, \mathrm{Q}_{1}, \mathrm{Sq}^{2}+\rho \mathrm{Sq}^{1}, \tau \mathrm{Sq}^{1}, \tau\right) & j \equiv 3 \bmod 4 .\end{cases}
$$

Here the ith component of $\mathrm{d}^{1}\left(\mathrm{cKW}_{2}\right)(q, j)$ is a map $\Sigma^{q+j, q} \mathbf{M} \mathbb{Z} / 2 \rightarrow \Sigma^{q+j+i, q+1} \mathbf{M} \mathbb{Z} / 2$.

Theorem 6.4. The restriction of the slice $\mathrm{d}^{1}$-differential for $\mathrm{kq}_{2}$ to the summand $\Sigma^{q+j, q} \mathbf{M} \mathbb{Z} / 2$ of $\mathrm{s}_{q}\left(\mathrm{kq}_{2}\right)$ is given by

$$
\begin{array}{r}
\mathrm{d}^{1}\left(\mathrm{kq}_{2}\right)(q, j)= \begin{cases}\left(\mathrm{Sq}^{3} \mathrm{Sq}^{1}, 0, \mathrm{Sq}^{2}\right) & q>j \equiv 0 \bmod 4 \\
\left(\mathrm{Sq}^{3} \mathrm{Sq}^{1}, \mathrm{Q}_{1}, \mathrm{Sq}^{2}, \rho+\tau \mathrm{Sq}^{1}\right) & q>j \equiv 1 \bmod 4 \\
\left(\mathrm{Sq}^{3} \mathrm{Sq}^{1}, 0, \mathrm{Sq}^{2}+\rho \mathrm{Sq}^{1}, 0, \tau\right) & q>j \equiv 2 \bmod 4 \\
\left(\mathrm{Sq}^{3} \mathrm{Sq}^{1}, \mathrm{Q}_{1}, \mathrm{Sq}^{2}+\rho \mathrm{Sq}^{1}, \tau \mathrm{Sq}^{1}, \tau\right) & q>j \equiv 3 \bmod 4\end{cases} \\
\mathrm{d}^{1}\left(\mathrm{kq}_{2}\right)(q, q)= \begin{cases}\left(\mathrm{Sq}^{2} \mathrm{Sq}^{1}, \mathrm{Sq}^{2}+\rho \mathrm{Sq}^{1}\right) & q \equiv 0 \bmod 4 \\
\left(\mathrm{Q}_{1}, \mathrm{Sq}^{2}, \rho+\tau \mathrm{Sq}^{1}, 0\right) & q \equiv 1 \bmod 4 \\
\left(\mathrm{Sq}^{2} \mathrm{Sq}^{1}, \mathrm{Sq}^{2}+\rho \mathrm{Sq}^{1}, \tau \mathrm{Sq}^{1}, \tau\right) & q \equiv 2 \bmod 4 \\
\left(\mathrm{Q}_{1}, \mathrm{Sq}^{2}+\rho \mathrm{Sq}^{1}, \tau \mathrm{Sq}^{1}, \tau\right) & q \equiv 3 \bmod 4 .\end{cases}
\end{array}
$$

Here the ith component of $\mathrm{d}^{1}\left(\mathrm{kq}_{2}\right)(q, j)$ is a map $\Sigma^{q+j, q} \mathbf{M} \mathbb{Z} / 2 \rightarrow \Sigma^{q+j+i, q+1} \mathbf{M} \mathbb{Z} / 2$.

Note that 9, Theorems 4.24, 4.36] contain information about first slice differentials for $\mathrm{kq}_{2^{r}}$ and $\mathrm{cKW}_{2^{r}}$, which could be used to analyze the first slice differentials for $\mathrm{C}_{2^{r}}$.

Theorem 6.5. Let $n>0$, and let $g:=\operatorname{gcd}(2 n, 12)$. The slices of $\mathrm{C}_{n \mathrm{~h}}$ are given as follows, up to summands of simplicial suspension higher than $q+2$ for $q \geq 4$.

$\mathrm{s}_{0}\left(\mathrm{C}_{n \mathrm{~h}}\right)=\mathrm{s}_{0}(\mathbf{1}) / 2 n$

$\mathrm{s}_{1}\left(\mathrm{C}_{n \mathrm{~h}}\right)=\mathrm{s}_{1}(\mathbf{1}) \vee \Sigma^{1,0} \mathrm{~s}_{1} \mathbf{1}$

$\mathrm{s}_{2}\left(\mathbf{C}_{n \mathbf{h}}\right)=\Sigma^{2,2} \mathbf{M} \mathbb{Z} / 2\left\{\alpha_{1}^{2}\right\} \vee \Sigma^{3,2} \mathbf{M} \mathbb{Z} / 2\left\{\overline{\alpha_{1}^{2}}\right\} \vee \Sigma^{3,2} \mathbf{M} \mathbb{Z} / g\left\{\alpha_{2}\right\} \vee \Sigma^{4,2} \mathbf{M} \mathbb{Z} / g\left\{\overline{\alpha_{2}}\right\}$

$\mathrm{s}_{3}\left(\mathrm{C}_{n \mathrm{~h}}\right)=\mathrm{s}_{3}(\mathbf{1}) \vee \Sigma^{1,0} \mathrm{~s}_{3} \mathbf{1}$

$\mathrm{s}_{4}\left(\mathrm{C}_{n \mathbf{h}}\right)=\Sigma^{4,4} \mathbf{M} \mathbb{Z} / 2\left\{\alpha_{1}^{4}\right\} \vee \Sigma^{5,4} \mathbf{M} \mathbb{Z} / 2\left\{\overline{\alpha_{1}^{4}}\right\} \vee \Sigma^{6,4} \mathbf{M} \mathbb{Z} / 2\left\{\alpha_{1} \alpha_{3}\right\} \vee \Sigma^{6,4} \mathbf{M} \mathbb{Z} / 2\left\{\nu^{2}\right\} \vee \cdots$

$\mathrm{s}_{q}\left(\mathrm{C}_{n \mathrm{~h}}\right)=\Sigma^{q, q} \mathbf{M} \mathbb{Z} / 2\left\{\alpha_{1}^{q}\right\} \vee \Sigma^{q+1, q} \mathbf{M} \mathbb{Z} / 2\left\{\overline{\alpha_{1}^{q}}\right\} \vee \Sigma^{q+2, q} \mathbf{M} \mathbb{Z} / 2\left\{\alpha_{1}^{q-3} \alpha_{3}\right\} \vee \cdots$

The unit map $\mathbf{1} \rightarrow \mathrm{kq}$ induces a map $\mathrm{C}_{n h} \rightarrow \mathrm{kq}_{n h}$, which induces the identity map on the slice summands $\mathbf{M} \mathbb{Z} / 2 n, \Sigma^{q, q} \mathbf{M} \mathbb{Z} / 2\left\{\alpha_{1}^{q}\right\}, \Sigma^{q+1, q} \mathbf{M} \mathbb{Z} / 2\left\{\overline{\alpha_{1}^{q}}\right\}, \Sigma^{q+2, q} \mathbf{M} \mathbb{Z} / 2\left\{\alpha_{1}^{q-3} \alpha_{3}\right\}$, and $\Sigma^{q+3, q} \mathbf{M} \mathbb{Z} / 2\left\{\overline{\alpha_{1}^{q-3} \alpha_{3}}\right\}$, and the map

$$
\Sigma^{3,2} \mathbf{M} \mathbb{Z} / g \vee \Sigma^{4,2} \mathbf{M} \mathbb{Z} / g \stackrel{\left(\partial_{2 n}^{g} \quad \operatorname{inc}_{2 n}^{g}\right)}{\longrightarrow} \Sigma^{4,2} \mathbf{M} \mathbb{Z} / 2 n
$$

on summands of the two-slices.

Proof. The form of the slices follows from [10, Section 8] or [21, Theorem 2.12], since $\mathrm{h}$ induces multiplication by 2 on slices, and the slice functors are triangulated. The map $\mathrm{C}_{n \mathrm{~h}} \rightarrow \mathrm{kq}_{n \mathrm{~h}}$ is determined by the unit map $\mathbf{1} \rightarrow \mathrm{kq}$, whose behaviour on slices can be read off from [21, Lemmas 2.28, 2.29]. 
Theorem 6.6. The first slice differential has the following form on the given summands:

$$
\begin{aligned}
& \mathrm{d}^{1}\left(\mathrm{C}_{n \mathrm{~h}}\right)\left(\alpha_{1}^{0}\right)=\left(\mathrm{Sq}^{2} \partial_{2}^{2 n}, \mathrm{Sq}^{2} \mathrm{pr}_{2}^{2 n}\right) \\
& \mathrm{d}^{1}\left(\mathrm{C}_{n \mathrm{~h}}\right)\left(\alpha_{1}\right)= \begin{cases}\left(\partial_{g}^{2} \mathrm{Sq}^{2} \mathrm{Sq}^{1}, \mathrm{inc}_{g}^{2} \mathrm{Sq}^{2} \mathrm{Sq}^{1}, 0, \mathrm{Sq}^{2}\right) & n \equiv 0(4) \\
\left(0, \mathrm{inc}_{g}^{2} \mathrm{Sq}^{2} \mathrm{Sq}^{1}, 0, \mathrm{Sq}^{2}\right) & n \equiv 2(4) \\
\left(\partial_{g}^{2} \mathrm{Sq}^{2} \mathrm{Sq}^{1}, 0,0, \mathrm{Sq}^{2}\right) & n \equiv 1(2)\end{cases} \\
& \mathrm{d}^{1}\left(\mathrm{C}_{n \mathrm{~h}}\right)\left(\alpha_{1}^{q}\right)=\left(\mathrm{Sq}^{3} \mathrm{Sq}^{1}, 0, \mathrm{Sq}^{2}\right) \quad q \geq 2 \\
& \mathrm{~d}^{1}\left(\mathrm{C}_{n \mathrm{~h}}\right)\left(\overline{\alpha_{1}}\right)= \begin{cases}\left(\mathrm{inc}_{g}^{2} \mathrm{Sq}^{2} \mathrm{Sq}^{1}, 0, \mathrm{Sq}^{2}\right) & n \equiv 0(2) \\
\left(\mathrm{inc}_{g}^{2} \mathrm{Sq}^{2} \mathrm{Sq}^{1}, \mathrm{inc}_{g}^{2} \mathrm{Sq}^{2}, \mathrm{Sq}^{2}, \tau \mathrm{Sq}^{1}\right) & n \equiv 1(2)\end{cases} \\
& \mathrm{d}^{1}\left(\mathrm{C}_{n \mathrm{~h}}\right)\left(\overline{\alpha_{1}^{q}}\right)= \begin{cases}\left(\mathrm{Sq}^{3} \mathrm{Sq}^{1}, 0, \mathrm{Sq}^{2}\right) & n \equiv 0(2) \\
\left(\mathrm{Sq}^{3} \mathrm{Sq}^{1}, \mathrm{Q}_{1}, \mathrm{Sq}^{2}, \tau \mathrm{Sq}^{1}\right) & n \equiv 1(2)\end{cases} \\
& \mathrm{d}^{1}\left(\mathrm{C}_{n \mathrm{~h}}\right)\left(\alpha_{2}\right)=\left(0, \mathrm{Sq}^{2} \partial_{2}^{g}, 0, \tau \partial_{2}^{g}\right) \\
& \mathrm{d}^{1}\left(\mathrm{C}_{n \mathrm{~h}}\right)\left(\overline{\alpha_{2}}\right)= \begin{cases}\left(\mathrm{Sq}^{2} \partial_{2}^{g}, 0, \tau \partial_{2}^{g}, 0\right) & n \equiv 0(4) \\
\left(\mathrm{Sq}^{2} \partial_{2}^{g}, \mathrm{Sq}^{2} \mathrm{pr}_{2}^{g}, \tau \partial_{2}^{g}, \tau \mathrm{pr}_{2}^{g}\right) & n \neq \equiv(4)\end{cases} \\
& \mathrm{d}^{1}\left(\mathrm{C}_{n \mathrm{~h}}\right)\left(\alpha_{1}^{q-3} \alpha_{3}\right)=\left(\mathrm{Sq}^{3} \mathrm{Sq}^{1}, 0, \mathrm{Sq}^{2}+\rho \mathrm{Sq}^{1}, 0, \tau\right) \quad q \geq 3 \\
& \left.\mathrm{~d}^{1}\left(\mathrm{C}_{n \mathrm{~h}}\right) \overline{\left(\alpha_{1}^{q-3} \alpha_{3}\right.}\right)= \begin{cases}\left(\mathrm{Sq}^{3} \mathrm{Sq}^{1}, 0, \mathrm{Sq}^{2}+\rho \mathrm{Sq}^{1}, 0, \tau\right) & n \equiv 0(2) \\
\left(\mathrm{Sq}^{3} \mathrm{Sq}^{1}, \mathrm{Q}_{1}, \mathrm{Sq}^{2}+\rho \mathrm{Sq}^{1}, \tau \mathrm{Sq}^{1}+\rho, \tau\right) & n \equiv 1(2)\end{cases}
\end{aligned}
$$

Proof. Based on the form of the unit map, most parts of the first slice differential are determined by Theorem 6.2. More precisely, the differentials $\mathrm{s}_{0} \mathrm{C}_{n \mathrm{~h}} \rightarrow \Sigma^{1,0} \mathrm{~s}_{1} \mathrm{C}_{n \mathrm{~h}}$, $\mathrm{s}_{2} \mathrm{C}_{n \mathrm{~h}} \rightarrow \Sigma^{1,0} \mathrm{~s}_{3} \mathrm{C}_{n \mathrm{~h}}$ and the listed behaviour for $q \geq 2$ is determined by Theorem 6.2 The remaining identities follow from Adem relations and the property $\mathrm{d}^{2}=0$, as well as comparison with $\mathrm{d}^{1}(\mathbf{1})$.

Theorem 6.6 may be used for computations of $\pi_{1+(\star)} \mathrm{C}_{n \mathrm{~h}}$ and $\pi_{2+(\star)} \mathrm{C}_{n \mathrm{~h}}$. Compared with corresponding slice spectral sequence computations for $\mathbf{1}$, the absence of integral motivic cohomology groups may be viewed as an advantage. Concrete presentations for $H^{\star-k, \star}$ as $\mathbf{K}^{\mathrm{MW}}$-modules seem to be known only in very few cases, contrary to the $\mathbf{K}^{\mathrm{MW}}$-module $h^{\star-k, \star} \cong \mathbf{K}_{\star-k}^{\mathrm{MW}} /(\eta, 2)$.

\section{REFERENCES}

[1] A. Ananyevskiy, O. Röndigs, and P. A. Østvær, On very effective hermitian K-theory, Math. Z. (2019), 14 pages, https://doi.org/10.1007/s00209-019-02302-z.

[2] T. Bachmann, The generalized slices of Hermitian K-theory, J. Topol. 10 (2017), no. 4, $1124-1144$.

[3] M. G. Barratt, Track groups. II, Proc. London Math. Soc. (3) 5 (1955), 285-329. MR 0073179

[4] D. Dugger and D. C. Isaksen, Motivic Hopf elements and relations., New York J. Math. 19 (2013), 823-871 (English).

[5] Low-dimensional Milnor-Witt stems over $\mathbb{R}$, Ann. K-Theory 2 (2017), no. 2, 175210. MR 3590344

[6] B. Gheorghe, The motivic cofiber of $\tau$., Doc. Math. 23 (2018), 1077-1127 (English).

[7] B. Gray, Ring spectra, Proceedings of the Advanced Study Institute on Algebraic Topology (Aarhus Univ., Aarhus, 1970), Vol. I, Mat. Inst., Aarhus Univ., Aarhus, 1970, pp. 143-153. Various Publ. Ser., No. 13. MR 0343271

[8] B. J. Guillou and D. C. Isaksen, The $\eta$-inverted $\mathbb{R}$-motivic sphere, Algebr. Geom. Topol. 16 (2016), no. 5, 3005-3027. MR 3572357 
[9] J. Kylling, O. Röndigs, and P. A. Østvær, Hermitian K-theory, Dedekind $\zeta$-functions, and quadratic forms over rings of integers in number fields, arXiv:1811.03940.

[10] M. Levine, A comparison of motivic and classical stable homotopy theories, J. Topol. 7 (2014), no. 2, 327-362. MR 3217623

[11] L. Mantovani, Localizations and completions in motivic homotopy theory, Dissertation, Universität Duisburg-Essen, 2018.

[12] J. Milnor, Algebraic K-theory and quadratic forms, Invent. Math. 9 (1969/1970), 318-344. MR 0260844 (41 \#5465)

[13] _ Introduction to algebraic K-theory, Princeton University Press, Princeton, N.J.; University of Tokyo Press, Tokyo, 1971, Annals of Mathematics Studies, No. 72. MR 0349811

[14] F. Morel, On the motivic $\pi_{0}$ of the sphere spectrum, Axiomatic, enriched and motivic homotopy theory, NATO Sci. Ser. II Math. Phys. Chem., vol. 131, Kluwer Acad. Publ., Dordrecht, 2004, pp. 219-260. MR 2061856 (2005e:19002)

[15] —, The stable $\mathbb{A}^{1}$-connectivity theorems, $K$-Theory 35 (2005), no. 1-2, 1-68. MR 2240215 (2007d:14041)

[16] S. Oka, Multiplications on the Moore spectrum, Mem. Fac. Sci. Kyushu Univ. Ser. A 38 (1984), no. 2, 257-276. MR 760188 (85j:55019)

[17] O. Röndigs, On the $\eta$-inverted sphere, $K$-Theory, Tata Inst. Fundam. Res. Stud. Math., vol. 19, Tata Inst. Fund. Res., Mumbai, 2018, pp. 41-64.

[18] O. Röndigs and P. A. Østvær, Rigidity in motivic homotopy theory, Math. Ann. 341 (2008), no. 3, 651-675. MR 2399164

[19] Slices of hermitian K-theory and Milnor's conjecture on quadratic forms, Geom. Topol. 20 (2016), no. 2, 1157-1212. MR 3493102

[20] O. Röndigs, M. Spitzweck, and P. A. Østvær, The second stable homotopy groups of motivic spheres, In preparation.

[21] O. Röndigs, M. Spitzweck, and P. A. Østvær, The first stable homotopy groups of motivic spheres, Ann. of Math. (2) 189 (2019), no. 1, 1-74. MR 3898173

[22] H. Toda, Composition methods in homotopy groups of spheres, Annals of Mathematics Studies, No. 49, Princeton University Press, Princeton, N.J., 1962. MR 0143217

[23] V. Voevodsky, Reduced power operations in motivic cohomology, Publ. Math. Inst. Hautes Études Sci. (2003), no. 98, 1-57. MR 2031198 (2005b:14038a)

Institut für Mathematik, Universität Osnabrück, Germany

E-mail address: oliver.roendigs@uni-osnabrueck.de 